\title{
A New Value Picking Regularization Strategy-Application to the 3-D Electromagnetic Inverse Scattering Problem
}

\author{
Jürgen De Zaeytijd, Ann Franchois, Senior Member, IEEE, and Jean-Michel Geffrin
}

\begin{abstract}
The nonlinear electromagnetic inverse scattering problem of reconstructing a possibly quasi-piecewise constant inhomogeneous complex permittivity profile is solved by iterative minimization of a pixel-based data fit cost function. Because of the ill-posedness it is necessary to introduce some form of regularization. Many authors apply a smoothing constraint on the reconstructed permittivity profile, but such regularization smooths away sharp edges. In this paper, a simple yet effective regularization strategy, the value picking (VP) regularization, is proposed. This new technique is capable of reconstructing piecewise constant permittivity profiles without degrading the edges. It is based on the knowledge that only a few different permittivity values occur in such profiles, the values of which need not be known in advance. VP regularization does not impose this a priori information in a strict sense, such that it can be applied also to profiles that are only approximately piecewise constant. The VP regularization is introduced in the solution of the inverse problem by adding a choice function to the data fit cost function for every permittivity unknown in the discretized problem. When minimized, the choice function forces the corresponding permittivity unknown to be close to one member of a set of auxiliary variables, the VP values, which are continuously updated throughout the iterations. To minimize the regularized cost function, a half quadratic Gauss-Newton optimization technique is presented. Finally, a stepwise relaxed VP regularization scheme is proposed, in which the number of VP values is gradually increased. This scheme is tested with synthetic and measured scattering data, obtained from inhomogeneous 3-D targets, and is shown to achieve high reconstruction quality.
\end{abstract}

Index Terms-Electromagnetic scattering, inverse problems, microwave imaging, optimization methods, regularization.

\section{INTRODUCTION}

$\mathbf{L}$ IKE many inverse problems, the electromagnetic inverse scattering problem-in this paper, the reconstruction of the complex permittivity of a dielectric object from scattering data-is extremely ill-posed. Of the three criteria in Hadamard's definition of ill-posedness [1], nonuniqueness and instability are the two major burdens in this context. The first problem stems from the combination of a limited information content in the

Manuscript received May 21, 2008; revised September 18, 2008. Current version published April 08, 2009. The work of J. Zaeytijd was supported by a Research Assistant of the Fund for Scientific Research-Flanders (F.W.O. -Vlaanderen).

J. De Zaeytijd and A. Franchois are with the Department of Information Technology (INTEC), Ghent University, B-9000 Gent, Belgium (e-mail: jurgen.dezaeytijd@intec.ugent.be; ann.franchois@intec.ugent.be).

J.-M. Geffrin is with the Aix-Marseille Université, Ecole Centrale Marseille, CNRS, Institut Fresnel, Domaine universitaire de St. Jérôme, 13397 Marseille, France (e-mail: Jean-Michel.Geffrin@fresnel.fr).

Digital Object Identifier 10.1109/TAP.2009.2015823 data with a desire to reconstruct the complex permittivity profile with a high spatial resolution, which can introduce more reconstruction parameters than there are degrees of freedom in the data. The instability issue is the result of a low sensitivity of the scattered field to some fluctuations in the permittivity profile with high spatial frequency. Without precautions, such unwanted fluctuations can be amplified in the reconstruction, without noticeably degrading the data fit. To cope with these problems, a regularization strategy is indispensable. The purpose of regularization is to use some a priori information on the target to select a proper permittivity profile from the many that fit the data within the uncertainty introduced by noise.

Often, isotropic smoothing constraints are imposed on the permittivity, e.g., [2]-[5], but these tend to smear out the edges in case of possibly quasi-piecewise constant permittivity profiles. Regularization methods that allow edges in the reconstruction while still mitigating unwanted fluctuations in homogeneous regions have been developed for image processing and can be applied to the electromagnetic inverse scattering problem as well. Total variation (TV) regularization [6]-[9] is one example. It satisfies a set of unifying criteria, proposed by Charbonnier et al. [10], which determine wether a regularization function is edge preserving. Such edge preserving regularization methods have been used in microwave imaging [11], [12]. Also methods based on level set techniques are used in inverse scattering [13], [14]. Both approaches effectively allow for piecewise constant reconstructions, but achieve this in different ways. Edge preserving regularization, for example, penalizes pixel-topixel differences unless they are large enough, in which case it is assumed that they correspond to edges in the profile. There is no distinct threshold below which differences are smoothed out and above which they are allowed to exist, but rather there is a transitional region which is encoded in potential functions. Level set techniques on the other hand implicitly only optimize for permittivity profiles that consist of regions of constant permittivity and, therefore, naturally allow for edges in the profile. Both methods have been used with success, but some possible disadvantages may be noted. The definition of the potential functions in edge preserving regularization generally implies the choice of some free parameter which tunes the aforementioned transitional region, such as $\delta$ in the TV scheme of [8], $\beta$ in [7], $\delta$ in [10] and $\delta_{R}$ and $\delta_{I}$ in [12]. It appears to us that the use of level set methods in situations with more than two different permittivities is somewhat complicated.

In this paper, we propose a new regularization scheme, denoted value picking (VP) regularization. It combines simplicity 
of implementation with an ability to reconstruct piecewise constant, or approximately piecewise constant permittivity profiles. Moreover, there is no need to determine additional parameters apart from the regularization parameter. The idea is to provide a limited number of reference permittivity values, the VP values, from which the regularization has to pick one for each permittivity unknown in the inverse problem. The choice for particular VP values is made through the minimization of a choice function for every permittivity unknown, constrained by the simultaneous minimization of a classical least squares data fit cost function. This basic idea has also been explored in [15]-[17] for binary objects [15], [16] or for one extra permittivity value [17]. However, the choice function in the present paper is different from the one used in those previous works, there is no limitation on the number of permittivity values and we do not assume the VP values to be known in advance. Rather, they act as auxiliary variables which are also optimized for. Even the number of VP values is updated in the course of the iterations. Starting from a severe restriction with only one VP value, more VP values are gradually added in a stepwise relaxed VP (SRVP) regularization scheme until a number is reached which allows for both a good data fit and a reconstruction which is close to piecewise constant. The optimization is performed using a Gauss-Newton type method, which results from applying Newton's method after a linearization of the scattered electric field in each step of the iterative solution. The VP regularizing function is "less than quadratic" and can be elegantly incorporated in the Gauss-Newton algorithm through a sequence of quadratic approximations. This yields a simple half quadratic minimization algorithm, similar to the one in [10]. It will be shown that the VP regularization scheme yields accurate reconstructions. Further research is needed to find out how it compares with various types of edge preserving regularization, but an advantage may be that it relies on a totally different principle. Indeed, VP regularization does not operate on the spatial distribution of the permittivity. Instead it clusters permittivity values in the complex plane. Therefore, it might also be useful in cases where one applies a different, nonpixel-based parameterization of the permittivity. Moreover, the framework naturally allows for the incorporation of a priori knowledge on the permittivity values.

The paper is organized as follows. In Section II, the three-dimensional electromagnetic inverse scattering problem is briefly revisited, introducing some notations for the rest of the paper. The VP regularized cost function is proposed in Section III and its minimization for a fixed number of VP values is discussed in Section IV. Next, the stepwise relaxed VP regularization scheme is outlined in Section V and finally this algorithm is tested on both synthetic and real measurement data in Section VI. Section VII concludes the paper.

\section{The Electromagnetic InVERse Scattering Problem}

Consider one or more inhomogeneous dielectric objects, represented by the complex permittivity function $\epsilon(\boldsymbol{r})=\epsilon_{r}(\boldsymbol{r})-$ $j \sigma(\boldsymbol{r}) / \omega$, where $\epsilon_{r}(\boldsymbol{r})$ is the dielectric permittivity and $\sigma(\boldsymbol{r})$ is the conductivity in the position $\boldsymbol{r}$ in a 3-D investigation domain $\mathcal{D}$ and where $\omega=2 \pi f$ is the angular frequency. The scatterers are embedded in a homogeneous dielectric background with complex permittivity $\epsilon_{\mathrm{b}}$, i.e., $\epsilon(\boldsymbol{r})=\epsilon_{\mathrm{b}}$ for $\boldsymbol{r} \notin \mathcal{D}$. The purpose of the electromagnetic inverse scattering problem is to estimate the permittivity function $\epsilon(\boldsymbol{r})$ everywhere within $\mathcal{D}$ from scattering data. In this paper, we employ single frequency data, i.e., all fields show a $e^{j \omega t}$ time dependency. This time dependency will be omitted in the rest of the paper, as well as the implicitly assumed frequency dependency of the permittivity. To collect the scattering data, the investigation domain is illuminated with a number of dipole fields which, in absence of the scattering objects, are given by

$$
\boldsymbol{E}_{i}^{\mathrm{inc}}(\boldsymbol{r})=-j \omega \mu_{0} \boldsymbol{G}_{\mathrm{b}}\left(\boldsymbol{r}-\boldsymbol{r}_{i}\right) \cdot \hat{\boldsymbol{u}}_{i}
$$

where $\boldsymbol{r}_{i}$ and the unit vector $\hat{\boldsymbol{u}}_{i}$ denote the position and the orientation of the ith elementary dipole, respectively, $\mu_{0}$ is the permeability of vacuum and $\boldsymbol{G}_{\mathrm{b}}$ is the Green dyadic of the homogeneous background medium, i.e.

$$
\boldsymbol{G}_{\mathrm{b}}\left(\boldsymbol{r}-\boldsymbol{r}^{\prime}\right)=\left[\rrbracket+\frac{1}{k_{\mathrm{b}}^{2}} \nabla \nabla\right] \frac{e^{-j k_{\mathrm{b}}\left|\boldsymbol{r}-\boldsymbol{r}^{\prime}\right|}}{4 \pi\left|\boldsymbol{r}-\boldsymbol{r}^{\prime}\right|}
$$

where $k_{\mathrm{b}}=\omega \sqrt{\epsilon_{\mathrm{b}} \mu_{0}}$ is the wave number of the background

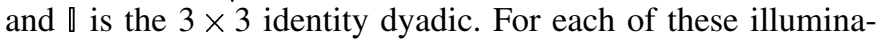
tions, the scattered electric field $\boldsymbol{E}_{i}^{\text {scat }}=\boldsymbol{E}_{i}-\boldsymbol{E}_{i}^{\text {inc }}$, defined as the difference between the total electric field in the presence of the scatterers and the the incident dipole field, is measured in a number of receiver points $\boldsymbol{r}_{j}$ along the directions $\hat{\boldsymbol{u}}_{j}$, i.e., the components $\boldsymbol{E}_{i}^{\text {scat }}\left(\boldsymbol{r}_{j}\right) \cdot \hat{\boldsymbol{u}}_{j}$ are measured. These data are collected in the $N^{D}$-dimensional vector $\boldsymbol{e}^{\text {meas }}$.

The least squares data fit in case of the electromagnetic inverse scattering problem is defined as

$$
\mathcal{F}^{\mathrm{LS}}(\varepsilon)=\frac{\left\|e^{\mathrm{scat}}(\varepsilon)-e^{\text {meas }}\right\|^{2}}{\left\|\boldsymbol{e}^{\text {meas }}\right\|^{2}}
$$

where $e^{\text {scat }}(\varepsilon)$ is a vector containing simulated scattered field data corresponding to a discretized permittivity profile, which is represented by the $N$-dimensional vector $\varepsilon$. The simplest discretization procedure for the permittivity is to cover $\mathcal{D}$ with a uniform cubic grid and to assume that the permittivity function is constant in each of the grid cells. In this case $N$ is the number of cells in the grid and $\varepsilon$ contains the values $\epsilon_{n}$ of the relative permittivity (i.e., $\epsilon / \epsilon_{0}$, with $\epsilon_{0}$ the permittivity of vacuum) in every cell of the grid. To calculate $e^{\text {scat }}(\varepsilon)$, a forward model is needed. For the results in this paper, an FFT-accelerated volume integral equation technique is employed, as described in [18]. Within this framework it is also easy to calculate derivatives of $\boldsymbol{e}^{\text {scat }}(\varepsilon)$ with respect to $\epsilon_{n}[5]$.

\section{VALUE PICKING REgULARIZATION}

Since electromagnetic fields, measured on any surface outside the source region, have a limited number of degrees of freedom in finite precision [19], the information content of the data vector is always limited and cannot be increased beyond a certain point by adding more transmitter and receiver positions. As a consequence, the minimum of $\mathcal{F}^{\mathrm{LS}}$ in (3) is generally not well-defined when a lot of optimization variables are considered. In the presence of noise on the data, several permittivity vectors may yield a data fit that is equal to or smaller than the data fit $\mathcal{F}^{\mathrm{LS}}\left(\varepsilon^{0}\right)$ obtained for the ideal permittivity vector $\varepsilon^{0}$, 


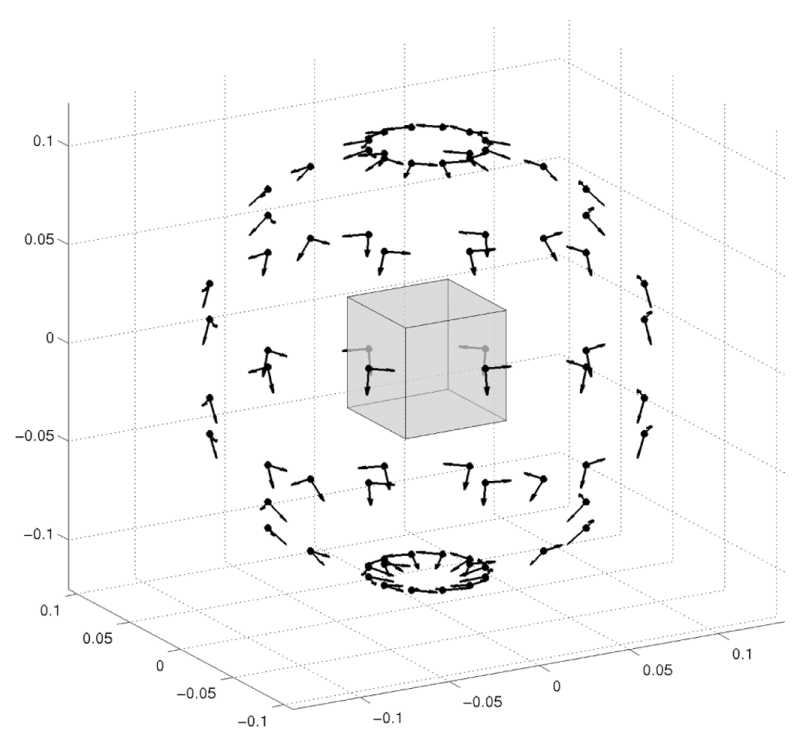

Fig. 1. A view of the dipole configuration and the investigation domain $\mathcal{D}$ which are used in the first reconstruction from simulated data. 144 dipoles are placed on a sphere with radius $11.2 \mathrm{~cm}$ and are oriented along the $\theta$ and $\phi$ directions. For each illumination, the scattered field is measured in all the dipole positions along the $\theta$ and $\phi$ directions.

i.e., the discretized permittivity which best approximates the actual permittivity function. We will denote this data fit $\mathcal{F}^{\mathrm{LS}}\left(\varepsilon^{0}\right)$ as the noise level $T^{N}$, a definition which accounts for both measurement noise and misfits due to the forward scattering model (discretization and numerical precision). The purpose of the regularization, as we define it in this paper, is to select a permittivity vector $\varepsilon$ close to $\varepsilon^{0}$ and for which $\mathcal{F}^{\mathrm{LS}}(\varepsilon) \approx T^{N}$ by using some restrictive property of $\varepsilon^{0}$. We exploit the property that $\varepsilon^{0}$ consists of only a few different permittivity values, the number of which is estimated to be smaller than or equal to $P$. This property is expected to be more restrictive for smaller $P$.

A regularized solution of the inverse problem is sought by incorporating this restrictive property into a regularized cost function

$$
\mathcal{F}(\varepsilon, c)=\mathcal{F}^{\mathrm{LS}}(\varepsilon)+\gamma \mathcal{F}^{P}(\varepsilon, \boldsymbol{c})
$$

where $\gamma$ is a positive regularization parameter. The Value Picking regularizing function $\mathcal{F}^{P}$ is given by

$$
\mathcal{F}^{P}(\varepsilon, \boldsymbol{c})=\frac{1}{N} \sum_{n=1}^{N} f^{P}\left(\left|\epsilon_{n}-c_{1}\right|^{2}, \ldots,\left|\epsilon_{n}-c_{P}\right|^{2}\right)
$$

where $f^{P}$ is the $P$-dimensional $(P \ll N)$ choice function and where the auxiliary variables $c_{p}$ are denoted the VP values. One of these VP values, more specifically $c_{P}$, is fixed to the known relative background permittivity, i.e., $c_{P}=\epsilon_{\mathrm{b}} / \epsilon_{0}$, and the $P-1$ other VP values are collected in the vector $\boldsymbol{c}$. The cost function is minimized for both $\varepsilon$ and $c$.

The choice function of dimension $P, f^{P}: \mathbb{R}_{+}^{P} \rightarrow \mathbb{R}_{+}\left(\mathbb{R}_{+}\right.$is the set of nonnegative real numbers), is defined as

$$
f^{P}\left(u_{1}, \ldots, u_{P}\right)=F^{P}\left(u_{1}, \ldots, u_{P} ; 0\right)
$$

where $F^{P}$ is defined and evaluated through the recursion formula

$$
\begin{aligned}
F^{P}\left(u_{1}, \ldots, u_{P} ; x\right)= & \left(u_{P}+x\right) \\
& \times \frac{F^{P-1}\left(u_{1}, \ldots, u_{P-1} ; x\right)}{F^{P-1}\left(u_{1}, \ldots, u_{P-1} ; u_{P}+x\right)}
\end{aligned}
$$

and

$$
F^{1}\left(u_{1} ; x\right)=u_{1}+x .
$$

For example, the cases with $P=1, P=2$ and $P=3$ yield

$$
\begin{aligned}
f^{1}\left(u_{1}\right) & =u_{1} \\
f^{2}\left(u_{1}, u_{2}\right) & =\frac{u_{1} u_{2}}{u_{1}+u_{2}} \\
f^{3}\left(u_{1}, u_{2}, u_{3}\right) & =\frac{u_{1} u_{2} u_{3}\left(u_{1}+u_{2}+u_{3}\right)}{\left(u_{1}+u_{2}\right)\left(u_{1}+u_{3}\right)\left(u_{2}+u_{3}\right)} .
\end{aligned}
$$

A list of relevant properties of the choice function and some auxiliary definitions are given in the Appendix.

As is readily seen from (9)-(11) and for general $P$ from property 2) of the Appendix, the VP function $\mathcal{F}^{P}$ is minimal when VP values $c_{p}$ can be found such that every optimization variable $\epsilon_{n}$ is equal to one of these VP values, i.e., $\mathcal{F}^{P}=0$ for permittivity profiles with at most $P$ different permittivity values. The minimization of (4) thus favors this kind of permittivity profiles. To our knowledge, the choice function (6)-(8) has not been used elsewhere. Its definition is the result of three major demands: (i) it should be zero if and only if one or more of its arguments are zero; (ii) it should be symmetric in its arguments; and (iii) for ease of use in optimization algorithms, its order should not increase for increasing $P$, i.e., the function should be bounded by a polynomial function with a fixed degree, independent of $P$. A function like $f^{P}=u_{1} \cdot u_{2} \ldots \cdot u_{P}$, as is used in [3], does not satisfy the third demand. Because of property 11), the function $f^{P}$, defined in (6)-(8), is less than linear and hence the function $\mathcal{F}^{P}$ is less than quadratic.

When approaching the minimum of (4) during the optimization, a specific VP value is picked from $\left\{c_{1}, \ldots, c_{P}\right\}$ for every permittivity unknown $\epsilon_{n}$ and the difference $\left|\epsilon_{n}-c_{p}\right|^{2}$ between the considered permittivity unknown and the chosen VP value $c_{p}$ is minimized. Thanks to the particular form of the choice function, these choices are made in an intuitively attractive way. To investigate this, the VP function (5) is rewritten using property 10):

$$
\mathcal{F}^{P}(\varepsilon, \boldsymbol{c})=\frac{1}{N} \sum_{n=1}^{N} \sum_{p=1}^{P} b_{p, n}^{P}(\varepsilon, \boldsymbol{c})\left|\epsilon_{n}-c_{p}\right|^{2}
$$

which can be seen as a weighted sum of the penalty functions $\left|\epsilon_{n}-c_{p}\right|^{2}$, where the weights $b_{p, n}^{P}$ are calculated with the weight functions defined in (52)

$$
b_{p, n}^{P}(\varepsilon, c)=B_{p}^{P}\left(\left|\epsilon_{n}-c_{1}\right|^{2}, \ldots,\left|\epsilon_{n}-c_{P}\right|^{2}\right) .
$$




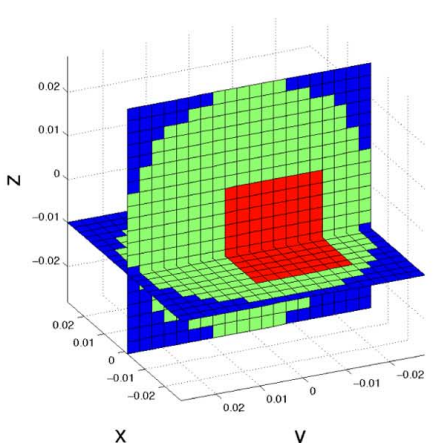

(a)

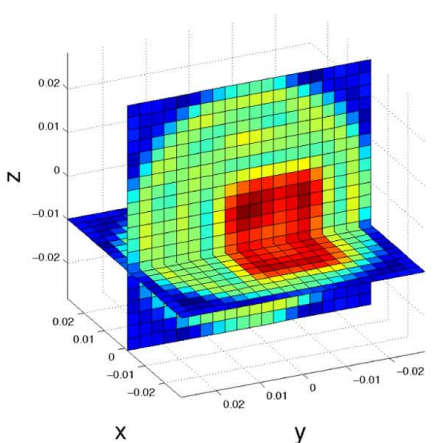

(c)

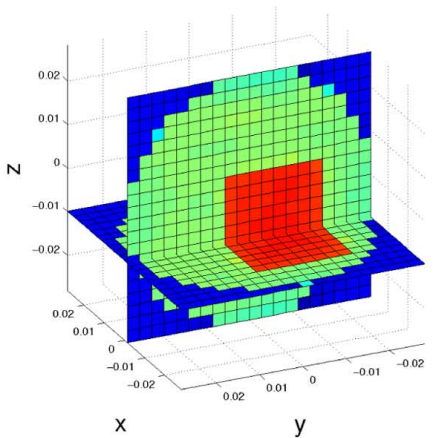

(e)

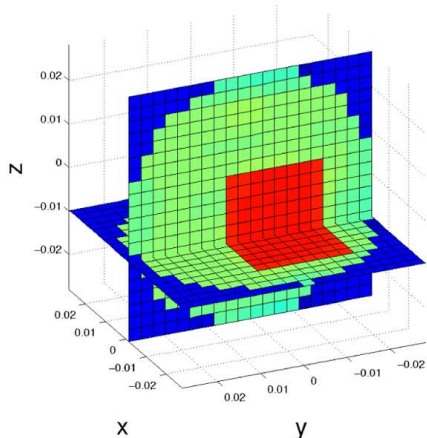

(g)
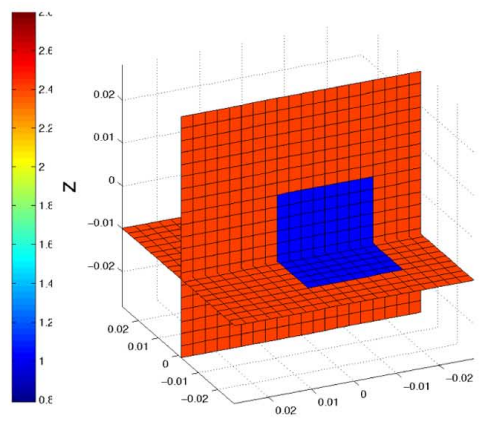

)
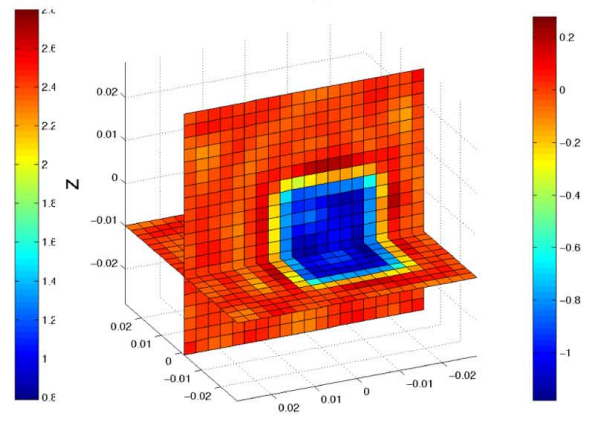

$x$

(d)
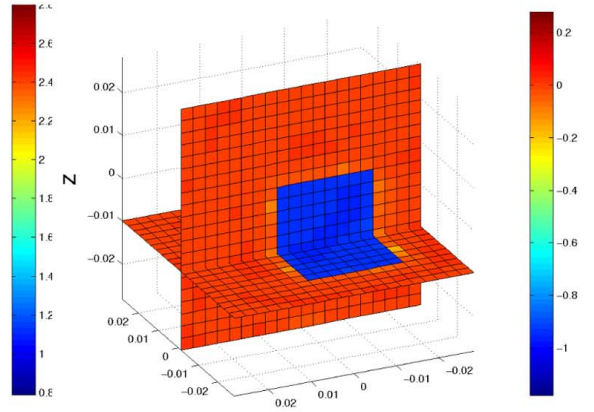

(f)
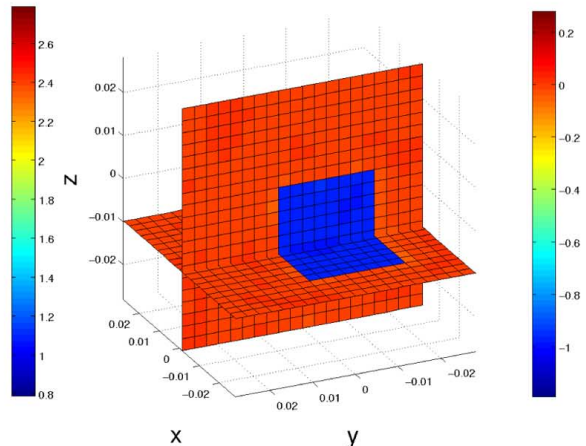

(h)

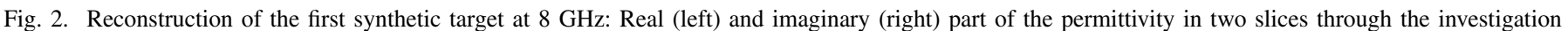

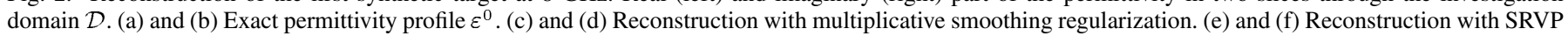
regularization with $P=3$. (g) and (h) Reconstruction with SRVP regularization with $P=4$.

The following observations can be made:

- Every time the difference $\left|\epsilon_{n}-c_{p}\right|$ between the permittivity unknown $\epsilon_{n}$ and the VP value $c_{p}$ becomes much larger than the differences $\left|\epsilon_{n}-c_{p^{\prime}}\right|$ between $\epsilon_{n}$ and the other VP values $c_{p^{\prime}}\left(p^{\prime} \neq p\right)$, the corresponding term $b_{p, n}^{P}\left|\epsilon_{n}-c_{p}\right|^{2}$ vanishes in (12). This is a direct result of property 4$)$. It also implies that the weight $b_{p, n}^{P}$ approaches zero and that $c_{p}$ does not contribute to the regularization of $\epsilon_{n}$.

- If $\left|\epsilon_{n}-c_{p}\right| \ll\left|\epsilon_{n}-c_{p^{\prime}}\right|, \forall p^{\prime} \neq p$, only the term $b_{p, n}^{P} \mid \epsilon_{n}-$ $\left.c_{p}\right|^{2}$ is retained in (12) and we have $b_{p, n}^{P} \approx b_{p, n}^{1} \stackrel{p, n}{=} 1$ and $b_{p^{\prime}, n}^{P} \approx 0$, for $p^{\prime} \neq p$, which is compatible with property 8 ). This means that the regularization will try to force $\epsilon_{n}=c_{p}$. 


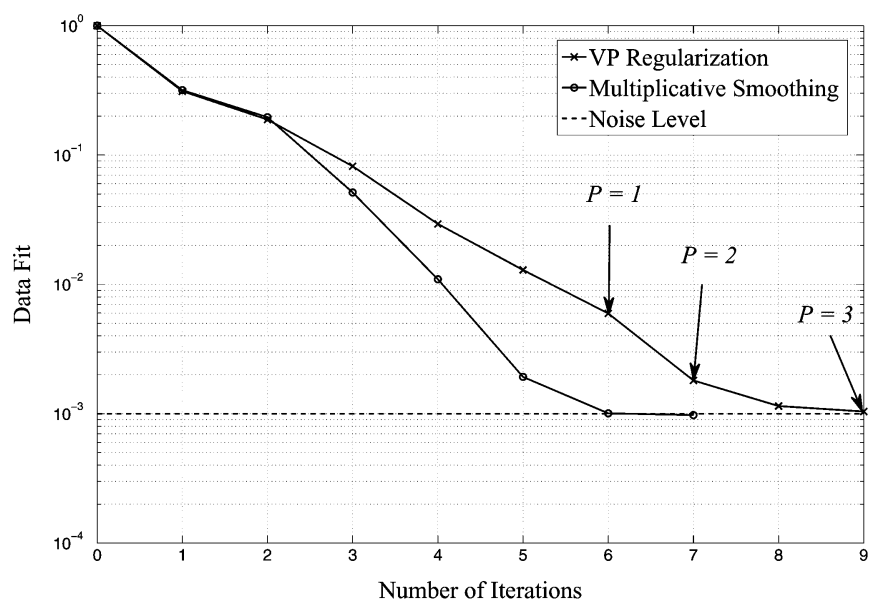

(a)

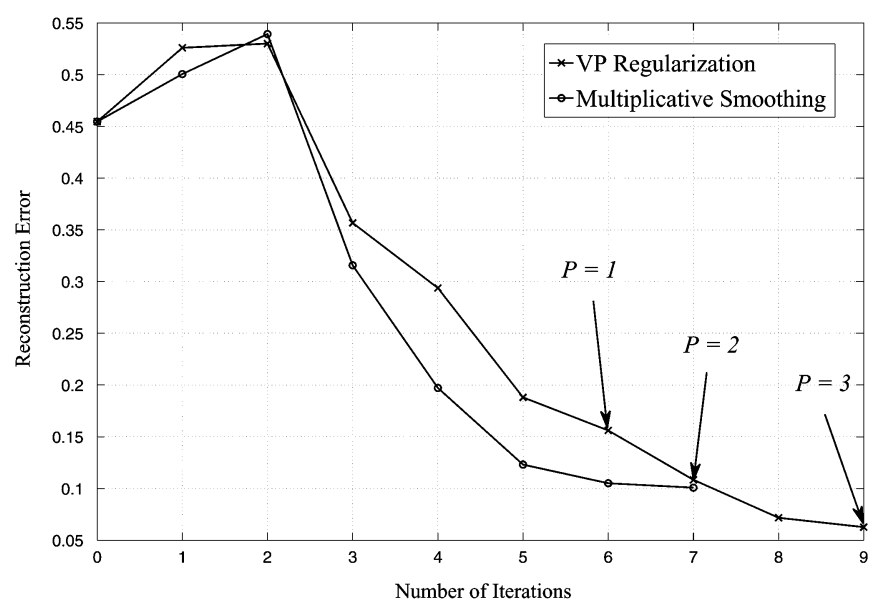

(b)

Fig. 3. The data fit cost function $\mathcal{F}^{\mathrm{LS}}$ (a) and the reconstruction error $\Delta \epsilon$ (b) versus the number of iterations during the reconstructions of the first synthetic target. The end of each step in the SRVP regularization scheme is indicated with an arrow. An extra VP value is added when $\left\|g^{Q}\right\| \leq \sqrt{2} \cdot 10^{-4}$ or when the data fit cost function $\mathcal{F}^{\mathrm{LS}}$ increases again.

- If $k$ differences $\left|\epsilon_{n}-c_{p}\right|$, for $p \in I \subset\{1, \ldots, P\}$, are of comparable size, but much smaller than the differences of $\epsilon_{n}$ with the other VP values, only $k$ terms $b_{p, n}^{k} \mid \epsilon_{n}-$ $\left.c_{p}\right|^{2}$ will remain in the summation (12) with weights $b_{p, n}^{k}$ somewhere between 0 and 1 . Moreover, it can be derived from property 5) that

$$
\sum_{p^{\prime} \in I} b_{p^{\prime}, n}^{k}\left|\epsilon_{n}-c_{p^{\prime}}\right|^{2} \leq\left|\epsilon_{n}-c_{p}\right|^{2}, \quad \forall p \in I
$$

which means that the sum of all the penalty terms corresponding to $\epsilon_{n}$ contributes less to (12) than a single penalty term with unit weight does. This way, the regularization will not force a decision too soon if the data fit does not provide enough driving force for it.

- It is possible that two or more VP values merge in the course of the minimization. $P$, thus, can be larger than the actual number of different permittivities.

Summarizing, whenever a permittivity variable becomes closer to one specific VP value than to the other VP values in course of the optimization, the regularization will try to force equality with this VP value. When there is no clear preference,

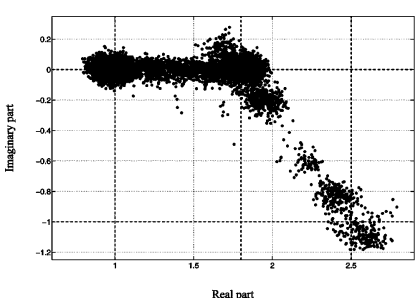

(a)

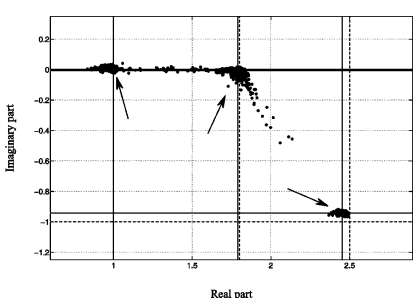

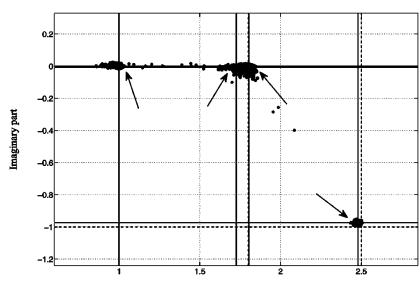

(c)

Fig. 4. Swarm plot of the $N$ complex permittivity values on the grid, represented with dots in the complex plane, for the reconstructions of the target of Fig. 2. Reconstructions: (a) of Fig. 2(c), (d) with multiplicative smoothing; (b) of Fig. 2(e), (f) with SRVP regularization and $P=3$; (c) of Fig. $2(\mathrm{~g})$ and (h) with SRVP regularization and $P=4$. The VP values are indicated as the intersections of the solid horizontal and vertical lines marked by arrows and the exact permittivity values lie at the nearby intersections of the dashed lines.

no choice will be made apart from disregarding VP values that are clearly far away from the considered optimization variable. The 3-D plot of the weights $b_{p, n}^{P}$ on the permittivity grid can be considered as a choice map, where values close to 1 indicate a choice for the VP value $c_{p}$ and values close to 0 indicate that $c_{p}$ has been disregarded in that position on the grid.

\section{The Minimization AlgORIthM}

The minimization of the cost function (4) is performed by alternately updating the permittivity profile and the VP values. To update the permittivity vector $\varepsilon$, an approximate line search [20] is performed along a modified Gauss-Newton descent direction. The VP values, subject to upper and lower bounds on real and imaginary parts, are then updated using a sequence of active set minimizations of quadratic functions that bound the VP function $\mathcal{F}^{P}$ from above.

\section{A. The Gauss-Newton Descent Direction for the Permittivity}

Starting from the permittivity profile $\varepsilon_{k}$ and the VP vector $c_{k}$ in iteration $k$ of the minimization process, a search direction $\boldsymbol{s}_{k}$ is calculated for $\varepsilon$, by applying a Gauss-Newton method. This search direction, however, is not directly computed from the cost function (4), but from a modified cost function

$$
\mathcal{F}^{Q}\left(\varepsilon, \boldsymbol{c} ; \varepsilon_{k}, \boldsymbol{c}_{k}\right)=\mathcal{F}^{\mathrm{LS}}(\varepsilon)+\gamma \mathcal{Q}^{P}\left(\varepsilon, \boldsymbol{c} ; \boldsymbol{\varepsilon}_{k}, \boldsymbol{c}_{k}\right)
$$

with

$$
\mathcal{Q}^{P}\left(\varepsilon, \boldsymbol{c} ; \varepsilon_{k}, \boldsymbol{c}_{k}\right)=\frac{1}{N} \sum_{n=1}^{N} \sum_{p=1}^{P} b_{p, n}^{P}\left(\varepsilon_{k}, \boldsymbol{c}_{k}\right)\left|\epsilon_{n}-c_{p}\right|^{2} .
$$

The difference between (16) and (12) [or between (15) and (4)] is that in (16) the weights $b_{p, n}^{P}$ are computed in $\left(\varepsilon_{k}, \boldsymbol{c}_{k}\right)$ and then kept fixed when $(\varepsilon, c)$ changes. Because of properties 10$)$ and 11), $\mathcal{Q}^{P}$ bounds $\mathcal{F}^{P}$ from above and touches with $\mathcal{F}^{P}$ in 


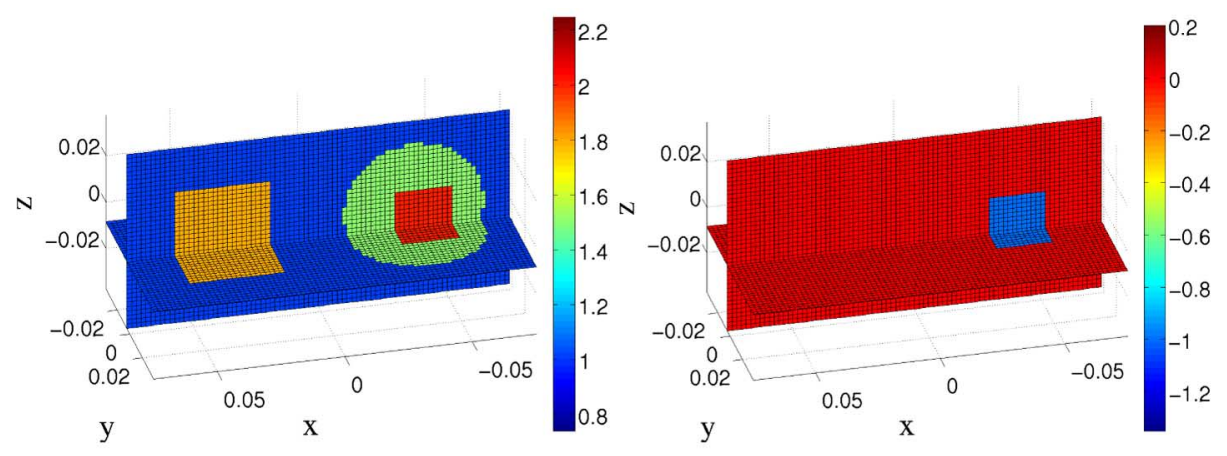

(a)

(b)

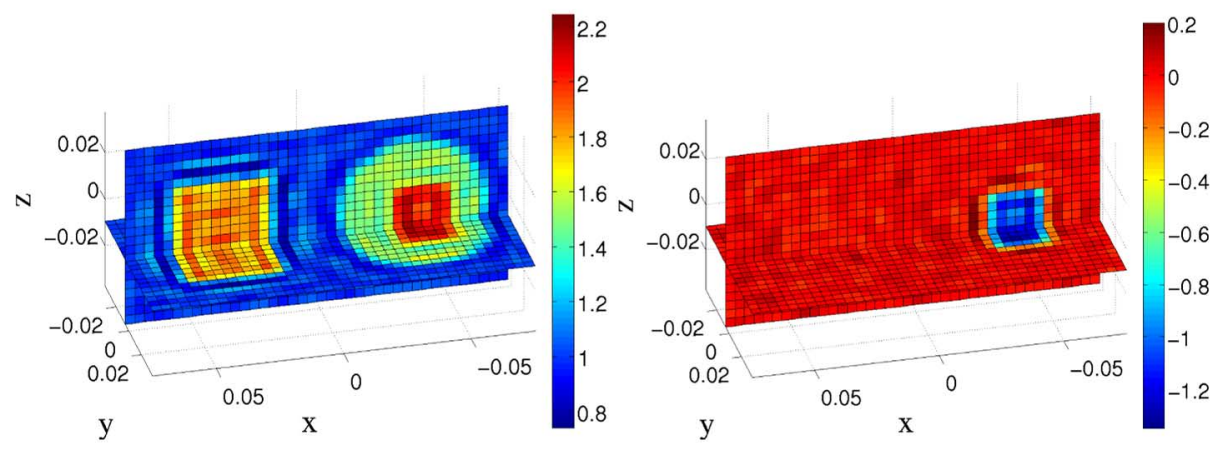

(c)

(d)

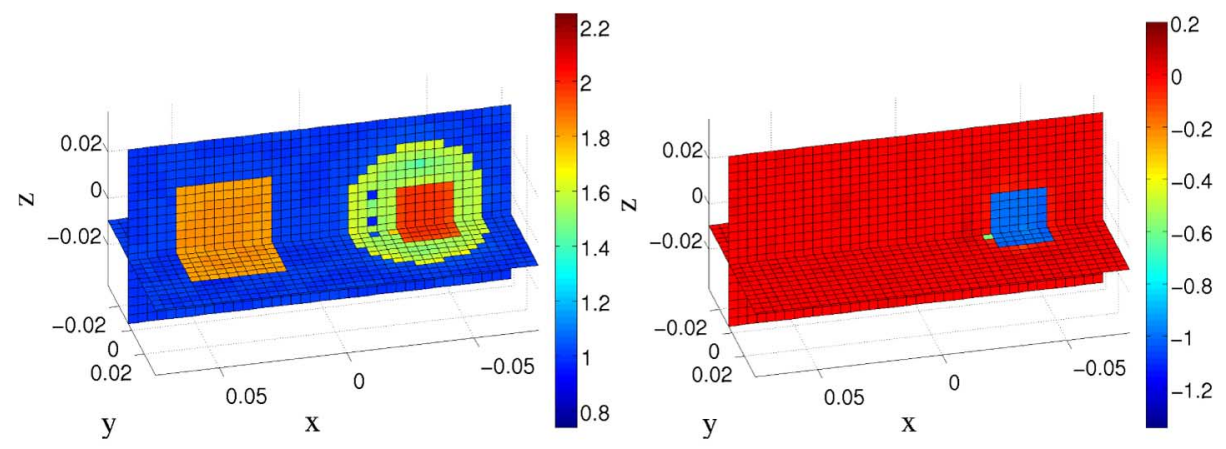

(e)

(f)

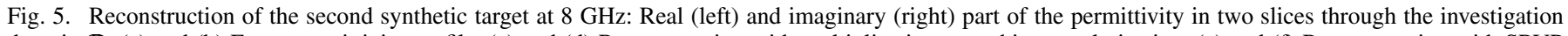

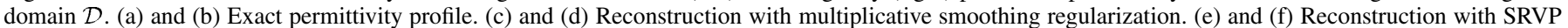
regularization and $P=4$.

$\left(\varepsilon_{k}, \boldsymbol{c}_{k}\right)$. Therefore, taking a reduction step in $\left(\varepsilon_{k}, \boldsymbol{c}_{k}\right)$ based on $\mathcal{F}^{Q}$ will also reduce $\mathcal{F}$. The use of (15) instead of (4) facilitates the incorporation of the VP regularization in the Gauss-Newton framework. As in [5], the complex optimization variables $\epsilon_{n}$ and their complex conjugate $\epsilon_{n}^{*}$ are considered as independent variables and the gradient $\boldsymbol{g}^{Q}$ and Hessian matrix $\boldsymbol{H}^{Q}$ of the real valued function $\mathcal{F}^{Q}$ are defined with respect to these variables

$$
\boldsymbol{g}^{Q}=\left[\begin{array}{c}
\frac{\partial \mathcal{F}^{Q}}{\partial \epsilon_{n}} \\
\frac{\partial \mathcal{F}^{Q}}{\partial \epsilon_{n}^{*}}
\end{array}\right], \quad \boldsymbol{H}^{Q}=\left[\begin{array}{cc}
\frac{\partial^{2} \mathcal{F}^{Q}}{\partial \epsilon_{n} \partial \epsilon_{m}} & \frac{\partial^{2} \mathcal{F}^{Q}}{\partial \epsilon_{n} \partial \epsilon_{m}^{*}} \\
\frac{\partial^{2} \mathcal{F}^{Q}}{\partial \epsilon_{n}^{2} \partial \epsilon_{m}} & \frac{\partial^{2} \mathcal{F}^{Q}}{\partial \epsilon_{n}^{*} \partial \epsilon_{m}^{*}}
\end{array}\right]
$$

They are given by

$$
\begin{aligned}
\boldsymbol{g}^{Q} & =\boldsymbol{g}^{\mathrm{LS}}+\gamma \boldsymbol{g}^{P} \\
\boldsymbol{H}^{Q} & =\boldsymbol{H}^{\mathrm{LS}}+\gamma \boldsymbol{H}^{P}
\end{aligned}
$$

where the gradient vector $\boldsymbol{g}^{\mathrm{LS}}$ and Hessian matrix $\boldsymbol{H}^{\mathrm{LS}}$ of the least squares data fit are given by

$$
\begin{aligned}
\boldsymbol{g}^{\mathrm{LS}} & =\frac{1}{\left\|\boldsymbol{e}^{\text {meas }}\right\|^{2}}\left[\begin{array}{c}
\boldsymbol{J}^{T}\left[\boldsymbol{e}^{\text {scat }}-\boldsymbol{e}^{\text {meas }}\right]^{*} \\
\boldsymbol{J}^{H}\left[\boldsymbol{e}^{\text {scat }}-\boldsymbol{e}^{\text {meas }}\right]
\end{array}\right] \\
\boldsymbol{H}^{\mathrm{LS}} & =\frac{1}{\left\|\boldsymbol{e}^{\text {meas }}\right\|^{2}}\left[\begin{array}{cc}
\boldsymbol{B} & \boldsymbol{J}^{T} \boldsymbol{J}^{*} \\
\boldsymbol{J}^{H} \boldsymbol{J} & \boldsymbol{B}^{*}
\end{array}\right] .
\end{aligned}
$$




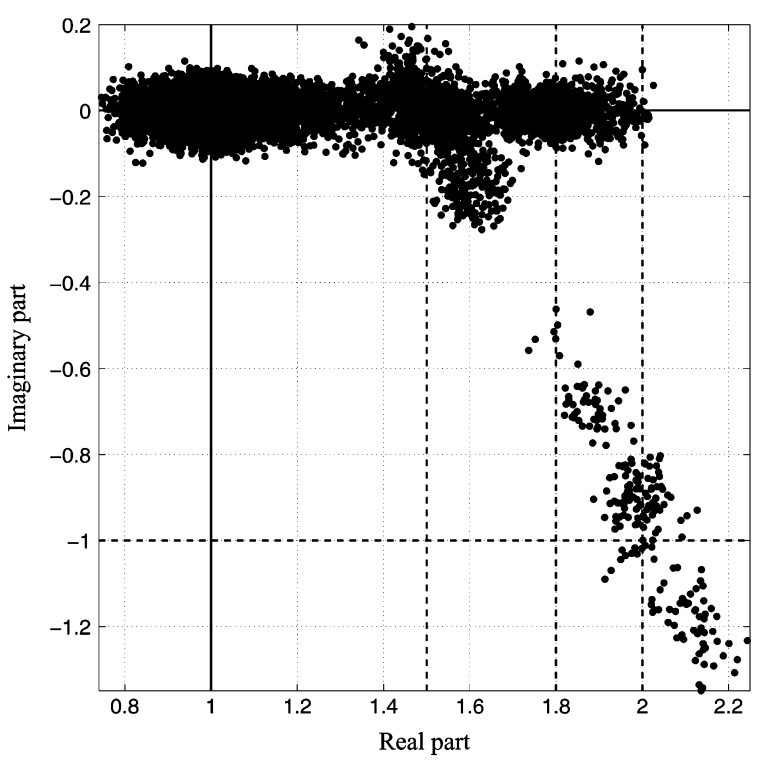

(a)

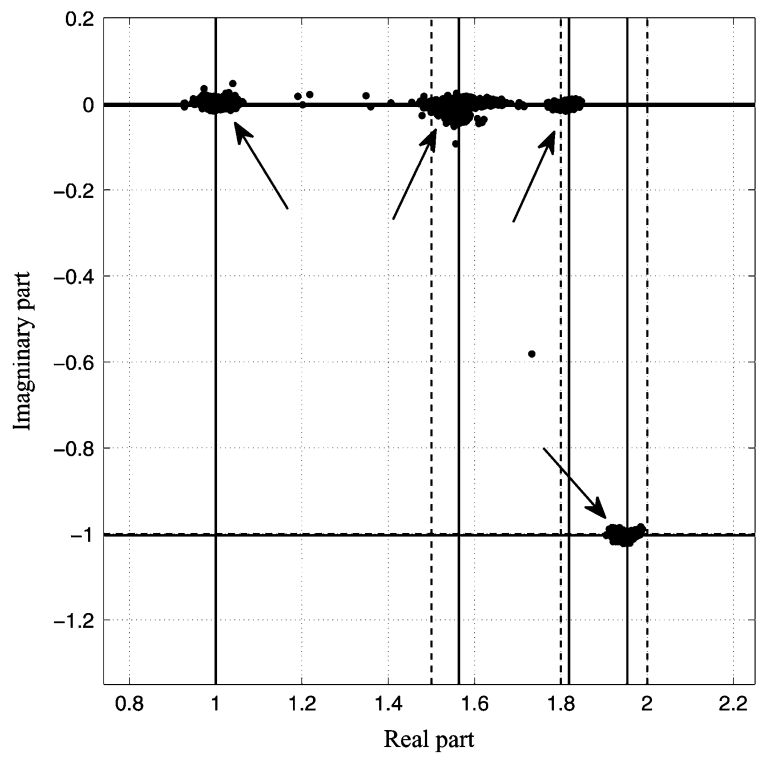

(b)

Fig. 6. Swarm plot of the $N$ complex permittivity values on the grid, represented with dots in the complex plane, for the reconstructions of the target of Fig. 5. Reconstructions: (a) of Fig. 5(c), (d) with multiplicative smoothing; (b) of Fig. 5(e), (f) with SRVP regularization. The VP values are indicated as the intersections of the solid horizontal and vertical lines marked by arrows and the exact permittivity values lie at the nearby intersections of the dashed lines.

The operations ${ }^{T}$ and ${ }^{H}$ stand for transpose and conjugate transpose, respectively. In these expressions, $\boldsymbol{J}$ is the $N^{\mathrm{D}} \times N$ Jacobian matrix, which contains the derivatives of the scattered field components with respect to the permittivity variables and the $N \times N$ matrix $\boldsymbol{B}$ contains products of second-order derivatives of the scattered field with the data residus:

$$
\begin{aligned}
{[\boldsymbol{J}]_{d n} } & =\frac{\partial\left[\boldsymbol{e}^{\mathrm{scat}}\right]_{d}}{\partial \epsilon_{n}} \\
{[\boldsymbol{B}]_{n m} } & =\left(\frac{\partial^{2} \boldsymbol{e}^{\mathrm{scat}}}{\partial \epsilon_{n} \partial \epsilon_{m}}\right)^{T}\left[e^{\mathrm{scat}}-\boldsymbol{e}^{\mathrm{meas}}\right]^{*}
\end{aligned}
$$

The gradient vector $\boldsymbol{g}^{P}$ and Hessian matrix $\boldsymbol{H}^{P}$ of the function $\mathcal{Q}^{P}$ are given by

$$
\begin{aligned}
\boldsymbol{g}^{P} & =\left[\begin{array}{c}
\boldsymbol{\Omega}^{P} \\
\left(\boldsymbol{\Omega}^{P}\right)^{*}
\end{array}\right] \\
\boldsymbol{H}^{P} & =\left[\begin{array}{cc}
\mathbf{0} & \boldsymbol{\Sigma}^{P} \\
\boldsymbol{\Sigma}^{P} & \mathbf{0}
\end{array}\right]
\end{aligned}
$$

with

$$
\begin{aligned}
{\left[\boldsymbol{\Omega}^{P}\right]_{n} } & =\frac{\partial \mathcal{Q}^{P}}{\partial \epsilon_{n}}\left(\varepsilon, \boldsymbol{c} ; \boldsymbol{c}_{k}, \boldsymbol{c}_{k}\right) \\
& =\frac{1}{N} \sum_{p=1}^{P} b_{p, n}^{P}\left(\varepsilon_{k}, \boldsymbol{c}_{k}\right)\left(\epsilon_{n}-c_{p}\right)^{*} \\
{\left[\boldsymbol{\Sigma}^{P}\right]_{n v} } & =\frac{\partial^{2} \mathcal{Q}^{P}}{\partial \epsilon_{n} \partial \epsilon_{v}^{*}}\left(\varepsilon, \boldsymbol{c} ; \varepsilon_{k}, \boldsymbol{c}_{k}\right) \\
& =\delta_{n, v} \frac{1}{N} \sum_{p=1}^{P} b_{p, n}^{P}\left(\varepsilon_{k}, \boldsymbol{c}_{k}\right) .
\end{aligned}
$$

Applying Newton's method yields an equation for the search direction $\boldsymbol{s}_{k}$ in iteration $k$

$$
\boldsymbol{H}_{k}^{Q}\left[\begin{array}{l}
\boldsymbol{s}_{k} \\
\boldsymbol{s}_{k}^{*}
\end{array}\right]=-\boldsymbol{g}_{k}^{Q}
$$

where the subscript $k$ indicates quantities evaluated in $(\varepsilon, \boldsymbol{c})=$ $\left(\varepsilon_{k}, \boldsymbol{c}_{k}\right)$. In the Gauss-Newton approximation, Newton's formula (28) is applied after a linearization of the scattered field $\boldsymbol{e}^{\text {scat }}$ as a function of $\boldsymbol{\varepsilon}$, i.e., the matrix $\boldsymbol{B}$ in (21) is neglected [5]. This yields the Gauss-Newton update system

$$
\begin{aligned}
\left(\boldsymbol{J}_{k}^{H} \boldsymbol{J}_{k}+\lambda^{2} \boldsymbol{\Sigma}_{k}^{P}\right) \boldsymbol{s}_{k} & \\
& =-\left(\boldsymbol{J}_{k}^{H}\left[\boldsymbol{e}_{k}^{\text {scat }}-\boldsymbol{e}^{\text {meas }}\right]+\lambda^{2}\left(\boldsymbol{\Omega}_{k}^{P}\right)^{*}\right)
\end{aligned}
$$

where the tradeoff parameter $\lambda^{2}$ is given by $\lambda^{2}=\gamma\left\|\boldsymbol{e}^{\text {meas }}\right\|^{2}$. It easily can be proven that if the matrix $\left(\boldsymbol{J}_{k}^{H} \boldsymbol{J}_{k}+\lambda^{2} \boldsymbol{\Sigma}_{k}^{P}\right)$ in the LHS of (29) is positive definite, then $\boldsymbol{s}_{k}$ is a descent direction with respect to both $\mathcal{F}^{Q}$ and $\mathcal{F}$. Since $\boldsymbol{J}_{k}^{H} \boldsymbol{J}_{k}$ is at least positive semi-definite, and since $\boldsymbol{\Sigma}_{k}^{P}$ is a diagonal matrix with strictly positive diagonal entries, this is clearly the case here. Moreover, towards the end of the minimization, when VP choices have been made for every permittivity variable, the matrix $\Sigma_{k}^{P}$ approaches $(1 / N) \boldsymbol{I}$, where $\boldsymbol{I}$ is the $N \times N$ unity matrix.

The search direction $\boldsymbol{s}_{k}$, obtained by the solution of (29), is used in an approximate line search [20], which determines $\beta$ such that

$$
\varepsilon_{k+1}=\varepsilon_{k}+\beta s_{k}
$$

is sufficiently close to the minimum of $\mathcal{F}$ along this line to ensure convergence of the descent method. Note that, although the search direction is derived from the function $\mathcal{F}^{Q}$, the line search along this direction is performed on the actual cost function $\mathcal{F}$. The recursive definitions (7) and (49) are used to evaluate the weight functions $B_{p}^{P}$ - which are needed for building the update system (29) and for computing the derivatives of $\mathcal{F}^{P}$ and 


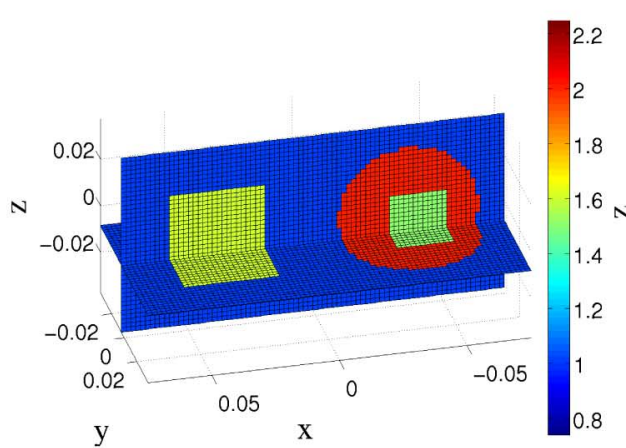

(a)

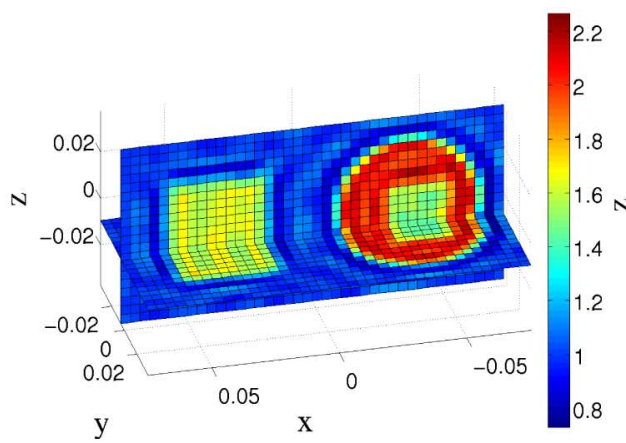

(c)

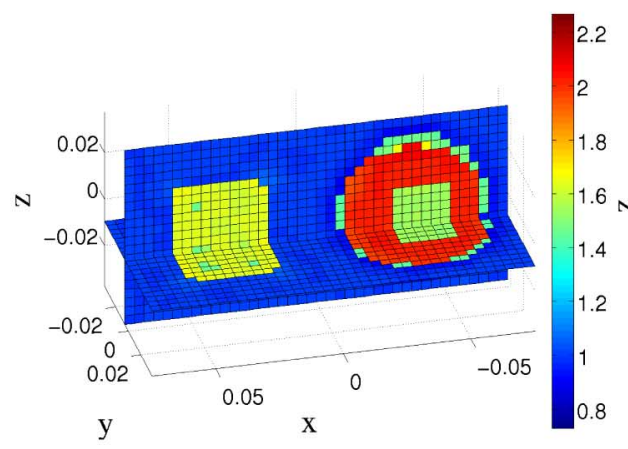

(e)

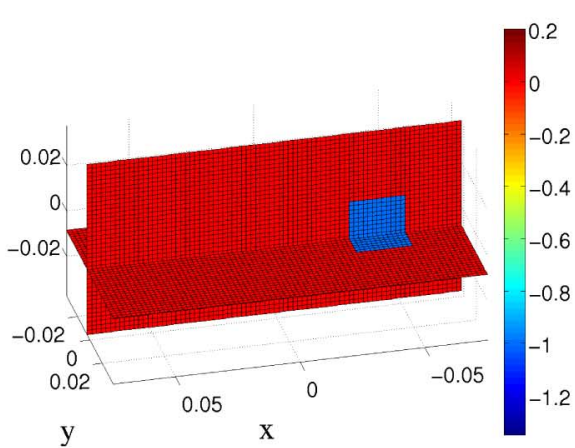

(b)

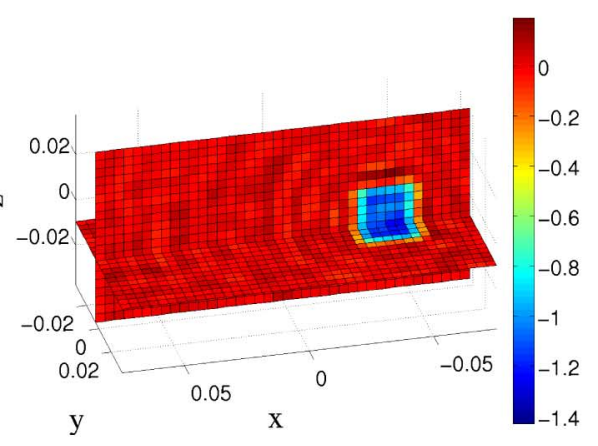

(d)

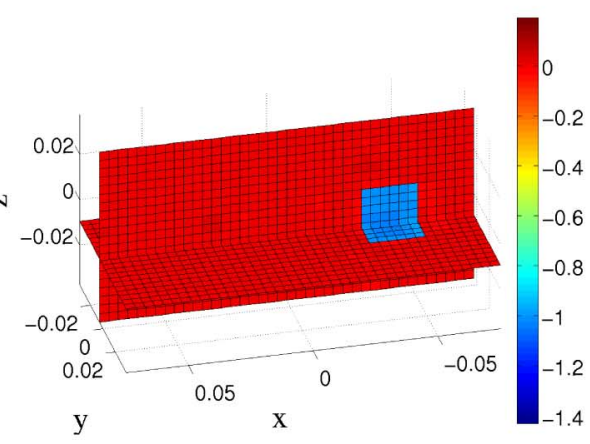

(f)

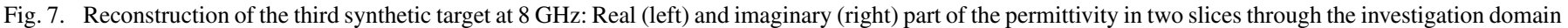

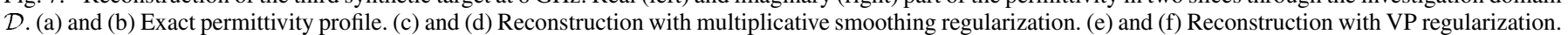

therefore of $f^{P}$ in the line search-and to evaluate the choice functions in $\mathcal{F}^{P}$, which is also needed in the line search.

\section{B. Updating the VP Values}

After updating $\varepsilon_{k}$ to $\varepsilon_{k+1}$ as described above, an optimization for $\boldsymbol{c}$ to obtain $\boldsymbol{c}_{k+1}$ is conducted. This step reduces the VP regularizing function in (4) without modifying the data fit $\mathcal{F}^{\mathrm{LS}}$. The optimization for $c$ is done by generating a sequence of VP vectors $\left\{\boldsymbol{c}_{\boldsymbol{l}}\right\}$, starting from $\boldsymbol{c}_{0}=\boldsymbol{c}_{k}$, where $\boldsymbol{c}_{\boldsymbol{l}+1}$ is the solution of the constrained quadratic minimization problem

$$
c_{l+1}=\underset{c}{\operatorname{argmin}} \mathcal{Q}^{P}\left(\varepsilon_{k+1}, c ; \varepsilon_{k+1}, c_{l}\right)
$$

subject to the upper and lower bounds

$$
\begin{aligned}
(p, r, u): \quad \Re\left(c_{p}\right) \leq c_{p, u}^{r}, \quad p=1, \ldots, P-1 \\
(p, r, l): \quad \Re\left(c_{p}\right) \geq c_{p, l}^{r}, \quad p=1, \ldots, P-1, \\
(p, i, u): \quad \Im\left(c_{p}\right) \leq c_{p, u}^{i}, \quad p=1, \ldots, P-1 \\
(p, i, l): \quad \Im\left(c_{p}\right) \geq c_{p, l}^{i}, \quad p=1, \ldots, P-1 .
\end{aligned}
$$

Since $\mathcal{Q}^{P}\left(\varepsilon_{k+1}, \boldsymbol{c} ; \boldsymbol{\varepsilon}_{k+1}, \boldsymbol{c}_{l}\right)$ touches $\mathcal{F}^{P}$ in the point $\left(\varepsilon_{k+1}, \boldsymbol{c}_{l}\right)$ and since $\mathcal{Q}^{P}\left(\varepsilon_{k+1}, \boldsymbol{c} ; \varepsilon_{k+1}, \boldsymbol{c}_{l}\right) \geq \mathcal{F}^{P}\left(\varepsilon_{k+1}, \boldsymbol{c}\right)$, every step in this scheme will reduce $\mathcal{F}^{P}$. The iterations are terminated for 


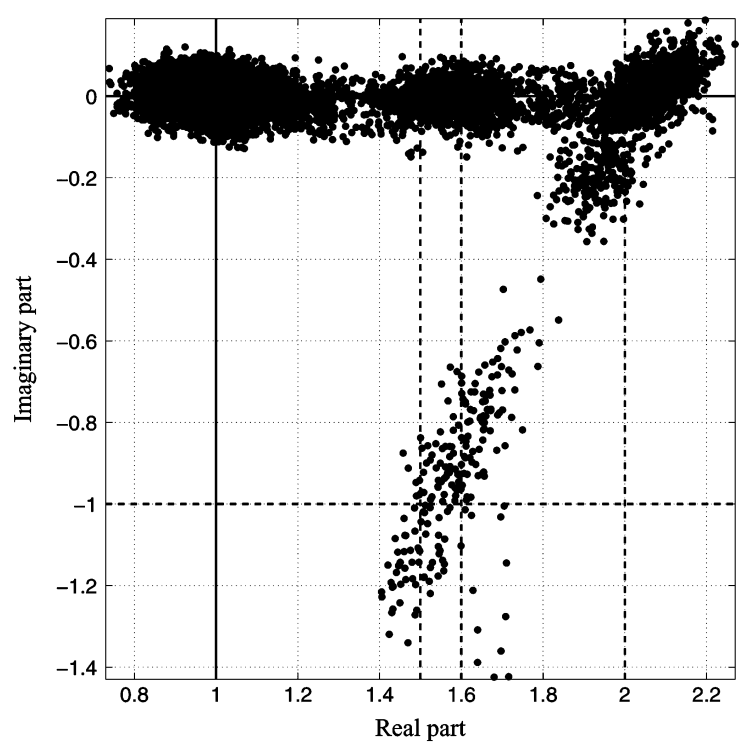

(a)

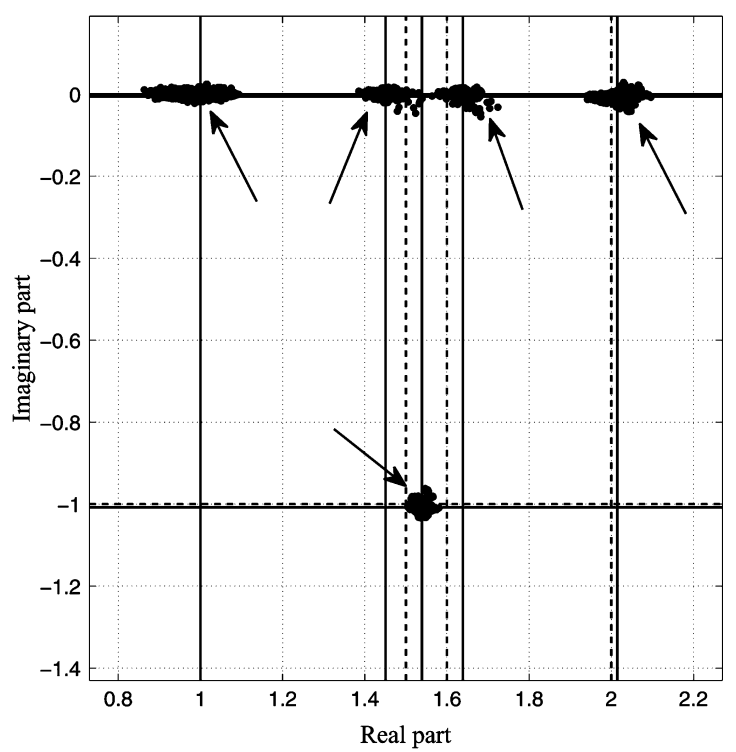

(b)

Fig. 8. Swarm plot of the $N$ complex permittivity values on the grid, represented with dots in the complex plane, for the reconstructions of the target of Fig. 7(a). Reconstructions: (a) of Fig. 7(c), (d) with multiplicative smoothing; (b) of Fig. 7(e), (f) with SRVP regularization. The VP values are indicated as the intersections of the solid horizontal and vertical lines marked by arrows and the exact permittivity values lie at the nearby intersections of the dashed lines.

$l=L$, such that $\left\|\boldsymbol{c}_{L}-\boldsymbol{c}_{L-1}\right\| \leq T^{C}$, some small threshold. We then conclude with $\boldsymbol{c}_{k+1}=\boldsymbol{c}_{L}$.

The minimization problem (31)-(35) for every $l$ is solved using a simple active set method which is a problem-specific reformulation of the general active set method for quadratic functions described in [20]. The iterations of this method generate a sequence $\left\{\boldsymbol{c}_{m}\right\}$ which starts from $\boldsymbol{c}_{0}=\boldsymbol{c}_{l}$ and the associated vector $\boldsymbol{\Lambda}_{l, 0}^{P}$ and matrix $\boldsymbol{\Xi}_{l}^{P}$, which for general $m$ are defined as

$$
\begin{aligned}
{\left[\boldsymbol{\Lambda}_{l, m}^{P}\right]_{p} } & =\frac{\partial \mathcal{Q}^{P}}{\partial c_{p}}\left(\varepsilon_{k+1}, \boldsymbol{c}_{m} ; \varepsilon_{k+1}, \boldsymbol{c}_{l}\right) \\
& =-\frac{1}{N} \sum_{n=1}^{N} b_{p, n}^{P}\left(\varepsilon_{k+1}, \boldsymbol{c}_{l}\right)\left(\epsilon_{k+1, n}-c_{m, p}\right)^{*}
\end{aligned}
$$

$$
\begin{aligned}
{\left[\boldsymbol{\Xi}_{l}^{P}\right]_{p q} } & =\frac{\partial^{2} \mathcal{Q}^{P}}{\partial c_{p} \partial c_{q}^{*}}\left(\varepsilon_{k+1}, \boldsymbol{c}_{m} ; \varepsilon_{k+1}, \boldsymbol{c}_{l}\right) \\
& =\delta_{p, q} \frac{1}{N} \sum_{n=1}^{N} b_{p, n}^{P}\left(\varepsilon_{k+1}, \boldsymbol{c}_{l}\right) .
\end{aligned}
$$

The iterations proceed as follows:

1) Determine the set of active constraints $I_{A}$. A constraint is said to be active if it yields an equality in (32)-(35).

2) Calculate $\Delta \boldsymbol{c}_{m}$ with

$$
\begin{aligned}
\Delta c_{m, p} & =-\frac{\left[\boldsymbol{\Lambda}_{l, m}^{P}\right]_{p}^{*}}{\left[\boldsymbol{\Xi}_{l}^{P}\right]_{p p}} \\
\Re\left(\Delta c_{m, p}\right) & =0, \quad \text { if } \quad(p, r, u) \in I_{A} \quad \text { or } \quad(p, r, l) \in I_{A} \\
\Im\left(\Delta c_{m, p}\right) & =0, \quad \text { if } \quad(p, i, u) \in I_{A} \quad \text { or } \quad(p, i, l) \in I_{A}
\end{aligned}
$$

where (39) and (40) possibly overwrite the result of (38).

3) Determine the smallest positive value of $\beta$, let it be $\beta_{\min }$, such that the line

$$
\boldsymbol{c}(\beta)=\boldsymbol{c}_{m}+\beta \Delta \boldsymbol{c}_{m}
$$

violates a presently inactive constraint and determine the corresponding constraint $(p, a, b)$, with $a=r$ or $a=i$ and $b=u$ or $b=l$.

4) If $\beta_{\min }<1$, put $\boldsymbol{c}_{m+1}=\boldsymbol{c}_{m}+\beta_{\min } \Delta \boldsymbol{c}_{m}$. Then calculate $\Lambda_{l, m+1}^{P}$ and add $(p, a, b)$ to $I_{A}$. Return to 2 .

5) If $\beta_{\min } \geq 1$ (including infinity), put $\boldsymbol{c}_{m+1}=\boldsymbol{c}_{m}+\Delta \boldsymbol{c}_{m}$ and calculate $\boldsymbol{\Lambda}_{l, m+1}^{P}$. Then, for every constraint in $I_{A}$, calculate the projection of $-\left(\boldsymbol{\Lambda}_{l, m+1}^{P}\right)^{*}$ on the direction in the complex plane which is perpendicular to that constraint and pointing outward from the constrained optimization domain. If the smallest of these projection values is positive or equal to zero, terminate with $\boldsymbol{c}_{\boldsymbol{l}+1}=\boldsymbol{c}_{m+1}$. Else, remove the corresponding constraint from $I_{A}$ and return to 2 .

Note that the VP values can be initialized at random within their constraints (32)-(35). The only limitation is that their initial values should not coincide. The presented algorithm treats identical VP values (with the same constraints) in an identical fashion, so once merged, two coinciding VP values will remain identical during the rest of the reconstruction.

\section{Further Analysis}

In this paper, the assumption that the desired permittivity vector $\varepsilon^{0}$ consists of only a few different permittivity values is used to regularize the inverse scattering problem and is not strictly imposed throughout the minimization, as is the case, for example, in inverse scattering algorithms using level set methods. As a result, the condition $\mathcal{F}^{P}=0$ generally is not satisfied exactly for the final reconstruction. Indeed, it is possible to prove the following statement [29]

$$
\nabla \mathcal{F}^{P}(\varepsilon, \boldsymbol{c})=0 \quad \Leftrightarrow \quad \mathcal{F}^{P}(\varepsilon, \boldsymbol{c})=0
$$

which means that a stationary point of $\mathcal{F}^{P}$ always is a global minimum of $\mathcal{F}^{P}$. Therefore, in a minimum of the cost function 


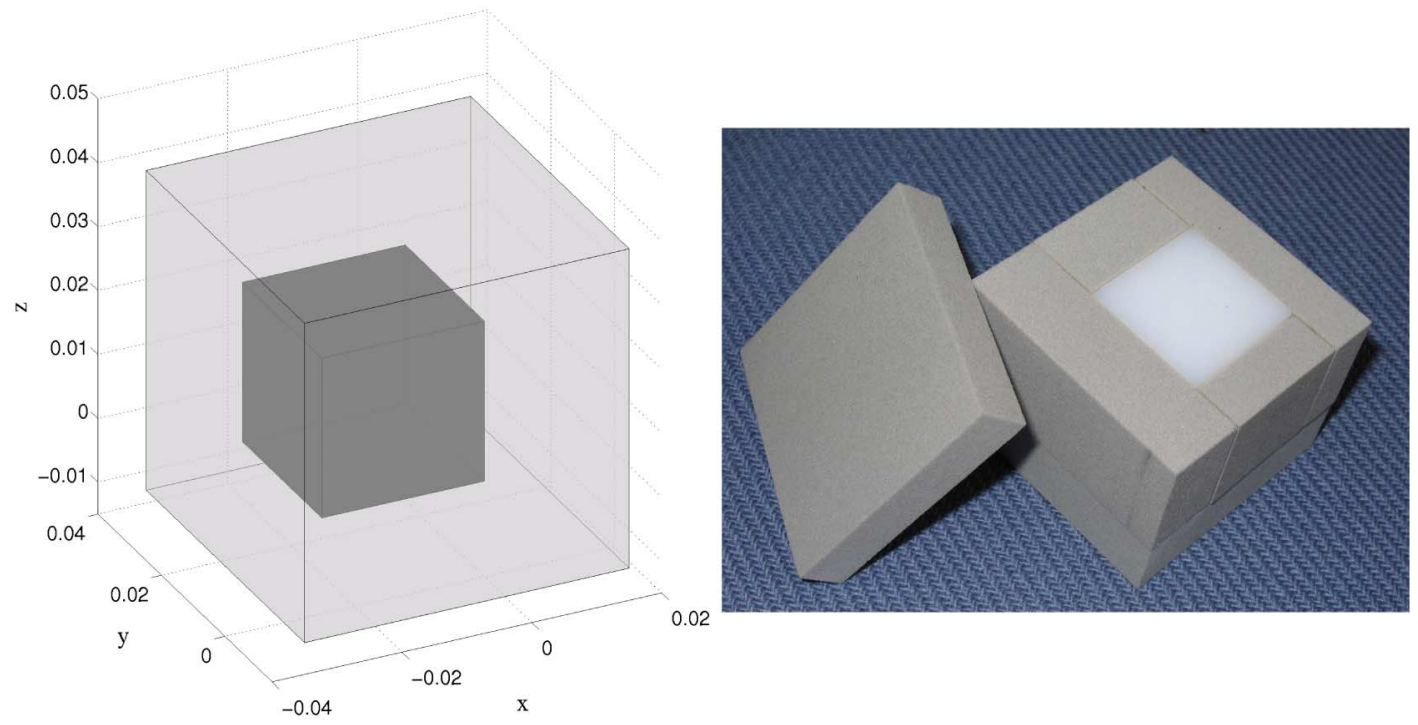

(a)

(b)

Fig. 9. The real world target used for the reconstruction from measurement data. A small cube with side $25 \mathrm{~mm}$ and permittivity $2.35 \epsilon_{0}$ is embedded in a larger cube with side $50 \mathrm{~mm}$ and permittivity $1.45 \epsilon_{0}$. Sketch (a) and a photograph of the actual target (b).

(4) where $\nabla \mathcal{F}=\nabla \mathcal{F}^{\mathrm{LS}}+\gamma \nabla \mathcal{F}^{P}=0$, there are two possibilities:

a) $\nabla \mathcal{F}^{P}=0$ and therefore $\nabla \mathcal{F}^{\mathrm{LS}}=0$ and $\mathcal{F}^{P}=0$.

b) $\nabla \mathcal{F}^{P} \neq 0$ and, therefore, $\nabla \mathcal{F}^{\mathrm{LS}} \neq 0$ and $\mathcal{F}^{P} \neq 0$.

Case a) implies that the minimum of the total cost function is also a minimum of the least squares data fit and exactly consists of at most $P$ different permittivity values. This is not very likely to happen when the data is noisy, especially with $P \ll N$. The resulting reconstruction, thus, is a tradeoff between data fit and the condition $\mathcal{F}^{P}=0$, as is expressed by case $\mathrm{b}$ ). As a result the permittivity unknowns will not perfectly coincide with the VP values, but will rather be clustered around those VP values in the complex plane.

\section{StePwise RElaxed VP REgularization}

The algorithm described in Section IV can become trapped in local minima above the noise level. Such spurious minima are likely to be introduced in the cost function since the VP regularizing function $\mathcal{F}^{P}$ has multiple (global) minimizers: every permittivity profile consisting of only $P$ different permittivity values that lie within the constraints imposed on $c$ yields $\mathcal{F}^{P}=$ 0 . To avoid this problem, one could use the VP regularization as a post processing step which starts from a reasonable initial estimate, obtained for example with the multiplicative smoothing regularization as described in [5] (a multiplicative regularization in the Gauss-Newton approach was introduced earlier in [21]). In such an approach, it is crucial that the data fit which corresponds to this initial estimate is sufficiently larger than the noise level such that the data fit cost function can guide the value picking process during the further minimization. It is then necessary to find a good criterion for switching from smoothing to VP regularization.

In this paper, we propose a different strategy. Let us first consider the extreme cases $P=N$ and $P=1$, where the optimization for $c$ is straightforward. The case $P=N$ yields $c_{n}=\epsilon_{n}$, for every $n$, which results in $\mathcal{F}^{P}=0, \Omega^{P}=0$ and $\boldsymbol{\Sigma}^{P}=(1 / N) \boldsymbol{I}$. As a result, the update system (29) is reduced to

$$
\left(\boldsymbol{J}_{k}^{H} \boldsymbol{J}_{k}+\frac{\lambda^{2}}{N} \boldsymbol{I}\right) \boldsymbol{s}_{k}=-\boldsymbol{J}_{k}^{H}\left[\boldsymbol{e}_{k}^{\mathrm{scat}}-e^{\mathrm{meas}}\right] .
$$

This update equation has been used by many authors, e.g., [22]-[24]. It results from minimizing the nonregularized least squares cost function $\mathcal{F}^{\mathrm{LS}}$ instead of (4) and applying a regularization only to the linear Gauss-Newton subproblems. This approach can be regarded as a Levenberg-Marquardt minimization algorithm [20], [24] applied to $\mathcal{F}^{\mathrm{LS}}$. Since the minimum of $\mathcal{F}^{\mathrm{LS}}$ generally is not well defined, the performance of this algorithm is significantly reduced in the presence of noise. We could say that this approach does not yield a regularized solution as defined in Section III. The case $P=1$ requires no optimization for $\boldsymbol{c}$, because the only VP value $c_{1}$ is kept fixed and equal to $\epsilon_{\mathrm{b}} / \epsilon_{0}$. It results in the cost function

$$
\mathcal{F}(\varepsilon, c)=\mathcal{F}^{\mathrm{LS}}(\varepsilon)+\frac{\gamma}{N}\left\|\varepsilon-\varepsilon_{\mathrm{b}}\right\|^{2}
$$

where the second term is a classical Tikhonov regularization [25] and where $\varepsilon_{\mathrm{b}}$ is the $N$-dimensional vector with $\left[\varepsilon_{\mathrm{b}}\right]_{n}=$ $\epsilon_{\mathrm{b}} / \epsilon_{0}, \forall n$. The corresponding update system is

$$
\left(\boldsymbol{J}_{k}^{H} \boldsymbol{J}_{k}+\frac{\lambda^{2}}{N} \boldsymbol{I}\right) \boldsymbol{s}_{k}=-\left(\boldsymbol{J}_{k}^{H}\left[\boldsymbol{e}_{k}^{\mathrm{scat}}-\boldsymbol{e}^{\text {meas }}\right]+\frac{\lambda^{2}}{N}\left(\varepsilon_{k}-\varepsilon_{\mathrm{b}}\right)\right) \text {. }
$$

The regularization (44) imposes a strong restriction on the permittivity vector $\varepsilon$. From these two extreme cases $P=1$ (strong restriction) and $P=N$ (no restriction) and from property 5), we conclude that increasing the number of VP values relaxes the regularization.

Since, according to the discrepancy principle, it is desirable to have as much regularization as possible without preventing 


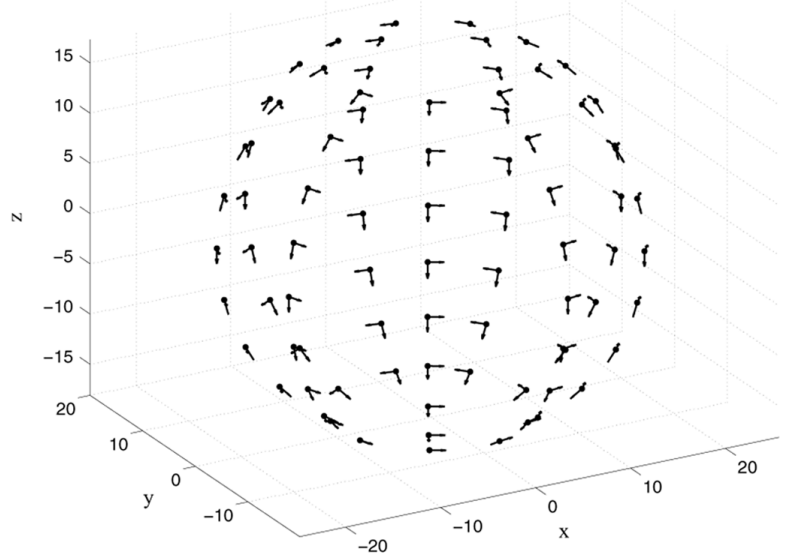

(a)

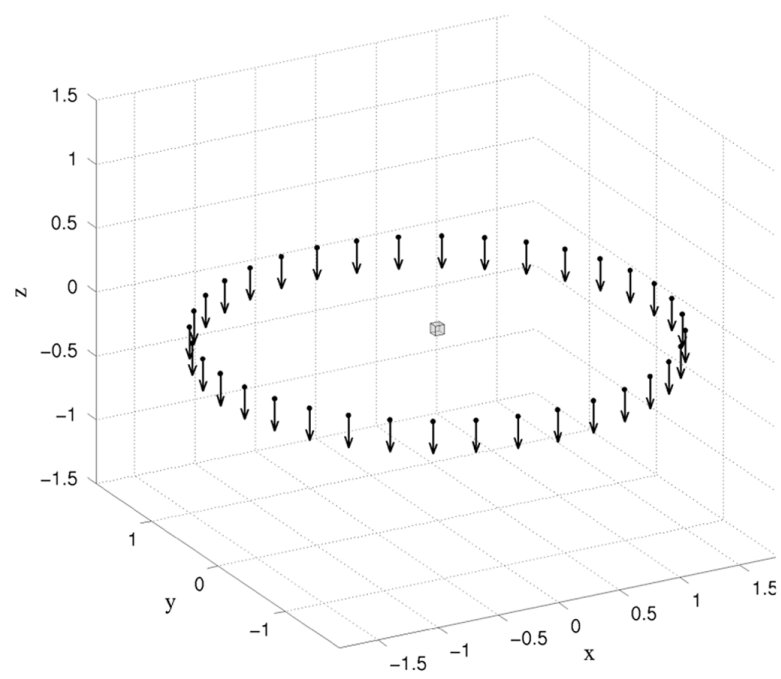

(b)

Fig. 10. The dipole configuration used for the reconstructions from measurement data. 162 transmitting dipoles are placed on a sphere with radius $20 \mathrm{~m}$ and are oriented along the $\theta$ and $\phi$ directions (a) and 36 receiving dipoles are placed on a circle with radius $1.796 \mathrm{~m}$ in the horizontal plane and are oriented along the negative $z$ axis (b). The investigation domain $\mathcal{D}$ is also depicted in (b).

the least squares data fit from reaching the noise level, we propose the following stepwise relaxed VP regularization (SRVP) strategy. The iterations start with $P=1$ and a fairly large value of the regularization parameter $\gamma$ and proceed until a minimum is reached, i.e., the gradient of the cost function is small enough, or until $\mathcal{F}^{\mathrm{LS}}$ increases again. The latter case implies that the VP regularization is making decisions that are not guided by the data fit. When $\gamma$ is large enough, this first step is terminated with $\mathcal{F}^{\mathrm{LS}}$ above the noise level. Then, the regularization is relaxed by adding an extra VP value and the optimization continues until the same stopping criterion is met. New VP values are added this way until $\mathcal{F}^{\mathrm{LS}}$ reaches the noise level or a threshold derived from an estimate of the noise level. Ofcourse, when the actual permittivity profile consists of $P_{0}$ different permittivity values, the algorithm should ideally reach the noise level when $P=P_{0}$. To this end $\gamma$ has to be chosen properly. When it is too large, the algorithm typically stops with $P>P_{0}$, since the large weight given to the regularization term then is compensated by adding more VP values, such that the regularization is sufficiently relaxed and allows for a data fit on the noise level. When it is too small, there is no strong restriction on the optimization and $\mathcal{F}^{\mathrm{LS}}$ will easily reach the noise level, even with a few VP values. The choice of $\gamma$ has been done by numerical experimentation so far. However, this does not require knowledge of the object. When the algorithm stops, i.e., when $\mathcal{F}^{\mathrm{LS}} \approx T^{N}$, we check for sufficient clustering of the permittivity unknowns around the VP values. We try to achieve this with as few VP values as possible. If the final reconstruction shows insufficient clustering, we restart with larger $\gamma$. If, on the other hand the clustering is sufficient, we try a smaller $\gamma$ too see if we can achieve a comparable clustering with fewer VP values. The addition of an extra VP value in every step of the SRVP scheme is performed by randomly initializing the VP value (making sure it does not coincide with already present VP values) and performing the minimization of Section IV-B before proceeding with the updates of the permittivity.

Finally, note that the SRVP regularization strategy can be seen as a minimization of the fixed cost function $\mathcal{F}=\mathcal{F}^{\mathrm{LS}}+\gamma \mathcal{F}^{N}$, where in a step with $P$ VP values $N-P$ VP values are frozen at infinity. This is a result of property 4$)$. In this perspective the cost function thus remains unaltered, but the optimization domain is restricted initially and gradually increased. We want to stress that the VP regularization assumes that a reconstruction is possible with $P \ll N$. If $P$ approaches $N$, there is no real regularization, as pointed out earlier.

\section{RESULTS}

To validate the proposed SRVP regularization algorithm, reconstructions from both synthetic and measured data are presented. The latter data were gathered in the bistatic polarimetric free-space measurement facility of Institut Fresnel, Marseille, France. More details about the measurement setup and methodology can be found in [26]-[28]. Throughout the rest of the section all targets are embedded in free space (i.e., $\epsilon_{b}=\epsilon_{0}$ ) and the operating frequency is $8 \mathrm{GHz}$, which yields a background wavelength $\lambda_{b}=0.0375 \mathrm{~m}$.

\section{A. Simulated Data}

The first target is a numerical phantom consisting of a cube with side $0.6 \lambda_{b}$ and permittivity $(2.5-j) \epsilon_{0}$, which is embedded in a sphere with radius $0.75 \lambda_{b}$ and permittivity $1.8 \epsilon_{0}$. The sphere and cube are centered at the origin and at the point $\left(-0.15 \lambda_{b},-0.15 \lambda_{b},-0.15 \lambda_{b}\right)$, respectively, in a reference system with axes parallel to the edges of the cube. The dipole configuration for the reconstruction of this target is depicted in Fig. 1. The dipole positions and orientations are indicated with dots and arrows, respectively. All 144 dipoles in the configuration are distributed over six meridional circles on a sphere with radius $3 \lambda_{b}$ that is centered at the origin. One half of the dipoles is oriented along the $\phi$ direction, while the other half is oriented along the $\theta$ direction. For this first example, each dipole is used to illuminate the target and the scattered field is measured in every dipole position and along each dipole direction. This yields a total of $N^{D}=20736$ data points. 


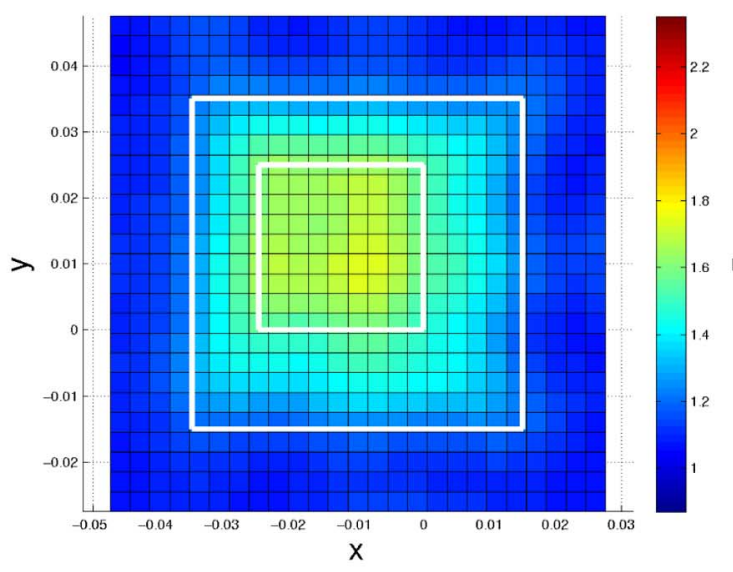

(a)

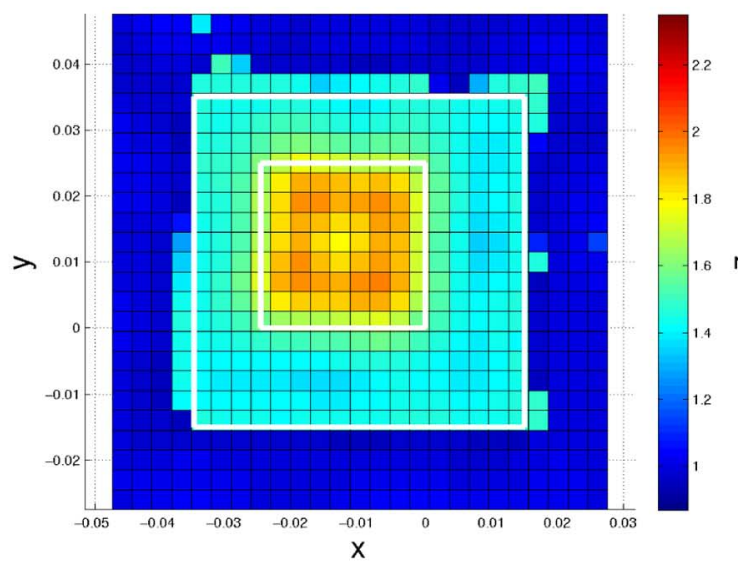

(c)

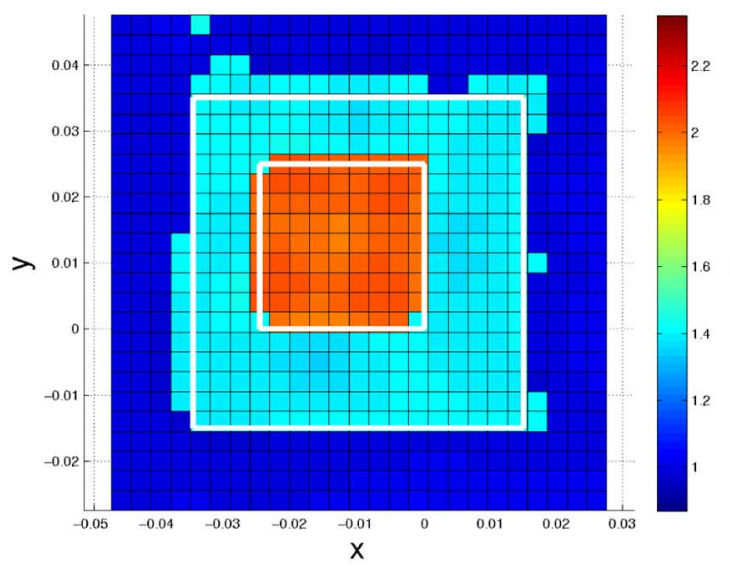

(e)

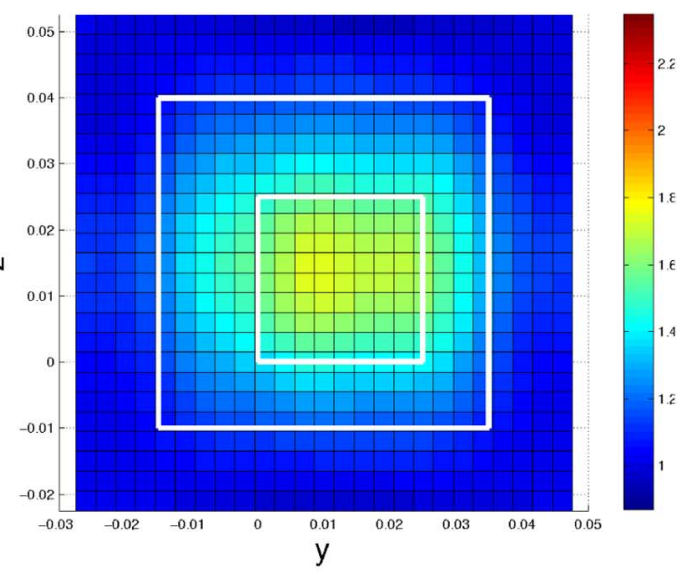

(b)

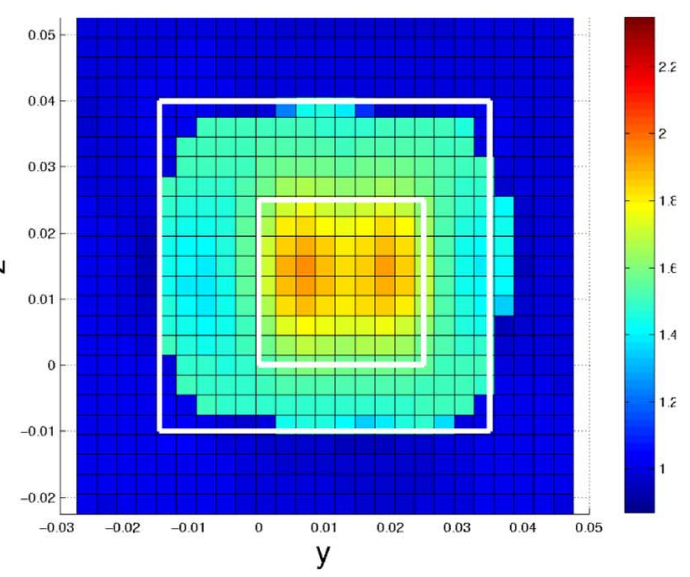

(d)

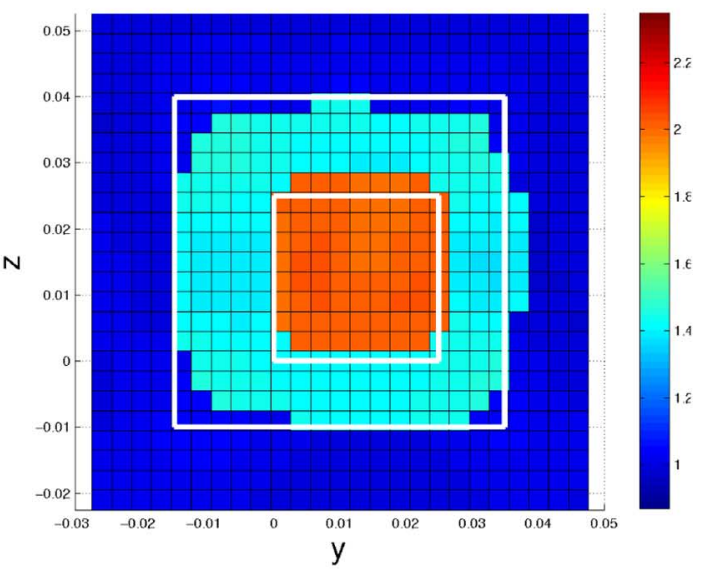

(f)

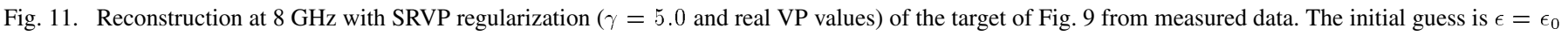

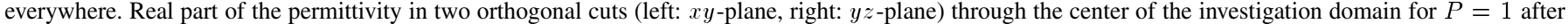
six iterations (a), (b), $P=2$ after three iterations (c), (d) and $P=3$ after eight iterations (e), (f). The solid white lines indicate the contours in the actual target.

The investigation domain $\mathcal{D}$ is a cube with side $1.5 \lambda_{b}$, centered at the origin and with edges parallel to the coordinate axes (Fig. 1), and the permittivity grid on this domain has a cell size $0.075 \lambda_{b}$, which yields 20 cells in each direction and thus a total of $N=8000$ permittivity unknowns. The synthetic data are obtained by solving the forward scattering problem with the same
FFT-accelerated volume integral equation technique as is used in the inverse scattering algorithm. Since the discretization grids for the fields and the permittivity in this forward simulation coincide with the grids used in the inversion algorithm, it is possible in principle to exactly reconstruct the target from these data and therefore this first reconstruction is a test under ideal cir- 
cumstances. Gaussian noise with an SNR of $30 \mathrm{~dB}$ is added to the data, which results in a noise level $T^{N}=10^{-3}$.

Fig. 2(a) and (b) shows the exact permittivity profile $\varepsilon^{0}$ in two slices through the investigation domain and the reconstructions of this profile are depicted in Fig. 2(c)-(h). For all these reconstructions, the optimization starts from the background permittivity in $\mathcal{D}$ using the following physical constraints on the VP values:

$$
\begin{aligned}
& \Re\left(c_{p}\right) \geq 1.0, \quad \forall p \\
& \Im\left(c_{p}\right) \leq 0.0, \quad \forall p .
\end{aligned}
$$

Fig. 2(c) and (d) shows a reconstruction with the multiplicative smoothing regularization of [5], which is obtained in seven iterations. One iteration comprises the calculation of a search direction, a line search for $\varepsilon$ and, in case of VP regularization, an update of the VP values. Fig. 2(e) and (f) shows the reconstruction with SRVP regularization $(\gamma=0.1)$ after the step with $P=3$ and 9 iterations in total. The iterations in each step of the SRVP scheme are terminated if $\left\|\boldsymbol{g}^{Q}\right\| \leq \sqrt{2} \cdot 10^{-4}$ or when the data fit increases again. The final reconstruction yields a data fit on the noise level, see Fig. 3(a), and a permittivity profile which is close to piecewise constant. Therefore, the algorithm can be terminated with $P=3$ with a result that is clearly very close to the actual permittivity profile (the final VP values are $\{2.45-0.94 j, 1.79-0.01 j, 1\})$. Fig. 3(b) shows the reconstruction error $\Delta \epsilon(\varepsilon)$, defined as

$$
\Delta \epsilon(\varepsilon)=\frac{\left\|\varepsilon-\varepsilon^{0}\right\|}{\left\|\varepsilon^{0}\right\|}
$$

which is smallest for the reconstruction with VP regularization.

When an extra VP value is added $(P=4)$, the result of Fig. $2(\mathrm{~g})$ and $(\mathrm{h})$ is obtained, which visually hardly can be distinguished from the result with $P=3$. The VP values now are $\{2.48-0.97 j, 1.80,1.73-0.01 j, 1\}$. The third VP value is close to the second one and mainly corresponds to cells along the surface of the sphere. The data fit for this result, which is obtained after only one additional iteration, has decreased negligibly (a reduction of $5 \cdot 10^{-4}$ ). This justifies a posteriori the termination with 3 VP values. Fig. 4 shows the "swarm plot" of the complex permittivity values in the complex permittivity plane for all the reconstructions of Fig. 2. The clustering in the reconstructions with VP regularization is apparent in these plots as opposed to the spreading of the permittivity values when using multiplicative smoothing.

Next, two more challenging targets are considered. The permittivity profiles are shown in Fig. 5(a) and (b) and in Fig. 7(a) and (b). The investigation domain is now a cuboid with dimensions $4 \lambda_{b} \times 2 \lambda_{b} \times 2 \lambda_{b}$ and there are three objects with different permittivities: a cube with side $0.6 \lambda_{b}$, embedded in a sphere with radius $0.75 \lambda_{b}$ and an additional cube with side $\lambda_{b}$. For the target of Fig. 5, the permittivities of these objects are $(2-j) \epsilon_{0}$, $1.5 \epsilon_{0}$ and $1.8 \epsilon_{0}$, respectively, and for the target of Fig. 7, they are $(1.5-j) \epsilon_{0}, 2 \epsilon_{0}$ and $1.6 \epsilon_{0}$, respectively. The dipole configuration is the same as the one used for the first example, except that the radius of the sphere on which the dipoles are located is now $4 \lambda_{b}$. Again, Gaussian noise with an SNR of $30 \mathrm{~dB}$ is added to the data and the grid for the data generation is now twice as fine as the inversion grid. The cell size of the inversion grid is $0.1 \lambda_{b}$ and the number of permittivity unknowns is $N=16000$.

The reconstructions with multiplicative smoothing and with SRVP regularization of the target of Fig. 5(a) and (b) are shown in Fig. 5(c) and (d) and (e) and (f), respectively. The VP regularization uses $\gamma=0.5$ and reaches the noise level with $P=4$ and 13 iterations. The final VP values are $\{1.95-1.00 j, 1.82,1.56,1.0\}$. Both reconstructions yield almost exactly the same data fit, but from the reconstructions and from the swarm plots of Fig. 6, it is clear that the VP reconstruction yields a better defined and more accurate estimation of the permittivity values and the shape of the objects. Due to the misfit between the grids for the data generation and the inversion algorithm, the sphere cannot be perfectly reconstructed and this apparently results in a slight overestimation of its permittivity and in the introduction of some stray cells with background permittivity inside it.

The reconstructions of the target of Fig. 7(a) and (b) are shown in Fig. 7(c)-(f). This time, the SRVP regularization, with $\gamma=1.0$, reaches the noise level with $P=5$ and 25 iterations. The final VP values are $\{2.01-0.01 j, 1.64,1.54-1.01 j, 1.45,1.0\}$. The extra VP value 1.45 apparently is added to define an intermediate permittivity level along the surface of the sphere. Lowering the regularization parameter, does not remedy this. Probably, the intermediate permittivity level is needed to compensate for the staircasing error in the coarser reconstruction grid. Indeed, no such intermediate level occurs around the larger cube in Fig. 5, which also has a relatively high permittivity. However, from Figs. 7 and 8, it can be concluded once more that the VP result provides more quantitative information on the original permittivity profile than the result obtained with multiplicative smoothing.

\section{B. Measured Data}

We finally consider a real world target that consists of a cube with side $0.67 \lambda_{b}$ and permittivity $2.35 \epsilon_{0}$, which is embedded in a larger cube with side $1.33 \lambda_{b}$ and permittivity $1.45 \epsilon_{0}$ (Fig. 9). Within the uncertainty introduced by positioning errors, the smaller cube is centered at the point $\left(-0.33 \lambda_{b}, 0.33 \lambda_{b}, 0.33 \lambda_{b}\right)$ and the larger cube at the point $\left(-0.27 \lambda_{b}, 0.27 \lambda_{b}, 0.40 \lambda_{b}\right)$ with respect to the center of the measurement facility of Institut Fresnel. In this bistatic measurement setup, the target is illuminated by a plane wave, generated by a parabolic antenna. In the forward model of our inverse solver, the source dipole is placed far from the target on a sphere with radius $20 \mathrm{~m}$ or $534 \lambda_{b}$, centered at the origin, to ensure that the incident field at the location of the target can be regarded as a plane wave. The measured scattered field has been normalized such that it corresponds to an incident field with amplitude 1 and zero phase at the origin. The 162 transmitting dipoles are placed on nine meridional circles, as depicted in Fig. 10(a). Again the dipole directions are evenly distributed over the $\theta$ and $\phi$ directions. The scattered field is collected in 36 points on a circle with radius $1.796 \mathrm{~m}$ or $47.9 \lambda_{b}$ in the horizontal plane and along the negative $z$-direction, as depicted in Fig. 10(b). Note that, due to technical limitations, only receivers that are further away than 
$50^{\circ}$ from the source meridian are used. The dimension of the data vector in this configuration is $N^{D}=4365$.

The investigation domain $\mathcal{D}$ for the reconstruction of the real world target is a cube with side $2.00 \lambda_{b}$, centered at $\left(-0.27 \lambda_{b}, 0.27 \lambda_{b}, 0.40 \lambda_{b}\right)$ and with edges parallel to the coordinate axes. The permittivity grid has a cell size of $0.08 \lambda_{b}$ $(=3 \mathrm{~mm})$, which results in 25 cells in each direction and a total of $N=15625$ permittivity unknowns. Considering the limited number of data, this inverse problem thus is heavily underdetermined, hence, without regularization, problems can arise with the uniqueness of the solution. Moreover, the update system (29) would be singular when $\lambda=0$. Indeed, the number of columns of the Jacobian matrix $\boldsymbol{J}$ is larger than the number of rows such that the rank of $\boldsymbol{J}^{H} \boldsymbol{J}$ certainly is smaller than its dimension. The VP regularization, however, apart from mitigating the effect of noise, also serves as a restriction on the number of degrees of freedom in the optimization space and the presented reconstruction will show that a good result can be obtained. As far as noise is concerned, there are the unavoidable measurement noise and also the discretization grid errors, especially since no attempt has been made to align the permittivity grid with the actual permittivity profile of the target, i.e., the faces in the permittivity grid do not coincide with the interfaces in the scatterer. From a number of reconstructions with the multiplicative smoothing regularization of [5] with different regularization parameters, the noise level is estimated as $T^{N} \approx 10^{-2}$.

The reconstruction shown in Fig. 11 is obtained with $\gamma=$ 5.0 , a rather large regularization parameter because of the high amount of noise on the data. To compensate for the high noise level and the fact that the system is underdetermined, some additional a priori information of the scatterer is employed: the VP values are kept real, satisfying the physical constraint $c_{p} \geq 1.0$. A new VP value is added each time the criterion $\left\|\boldsymbol{g}^{Q}\right\| \leq \sqrt{2} \cdot 10^{-4}$ is met or when the least squares data fit increases again. Starting from the background permittivity in $\mathcal{D}$, this yields 6 iterations with $P=1,3$ iterations with $P=2$ and 8 iterations with $P=3$. The position and dimensions of both cubes are quite accurately reconstructed within the resolution offered by the permittivity grid, although the inner cube is slightly too large. The reduced accuracy in the vertical direction is due to the antenna configuration, where the receiving antenna positions all are in the horizontal plane and where the transmitting dipoles are spaced further apart and span a smaller arc than the receiving dipoles. From the final VP values $\{1.42,2.01,1\}$ it can be concluded that the permittivity of the outer cube is accurately reconstructed and that the permittivity of the inner cube is a bit too low. However, the profile is almost piecewise constant and yields a data fit on the noise level, as can be seen from the swarm plot Fig. 12(a) and the data fit curves in Fig. 13.

The present example can be used to illustrate that the VP regularization allows for the introduction of a priori knowledge on the scatterer in an easy manner. Suppose we know that one of the permittivities in the profile is likely to be larger than 2 . The lower bound on the last added VP value then can be set to 2 . The result of this assumption is depicted in Fig. 14(a), (b). Again the data fit is on the noise level (Fig. 13) and the profile is close to piecewise homogeneous [Fig. 12(b)] with VP values $\{1.46,2.26,1\}$.

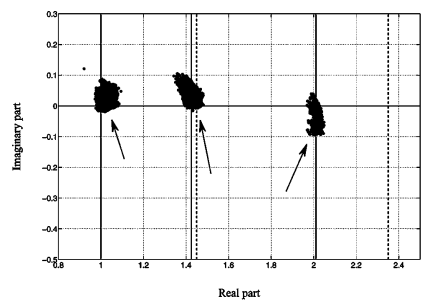

(a)

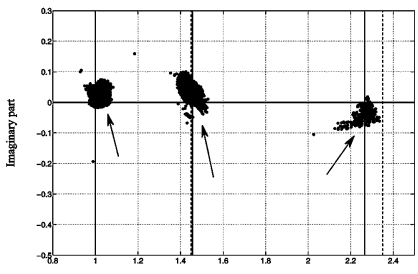

(b)

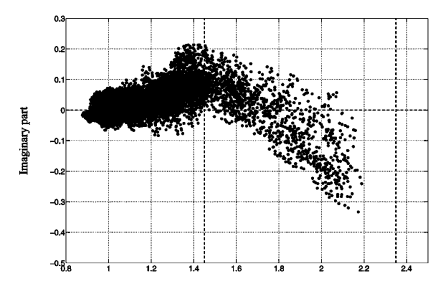

(c)

Fig. 12. Swarm plot of the $N$ complex permittivity values on the grid, represented with dots in the complex plane, for the reconstructions of the target of Fig. 9. Reconstructions: (a) of Fig. 11(e), (f) using SRVP regularization with real VP values; (b) of Fig. 14(a), (b) using SRVP regularization with real VP values and an extra lower bound of 2 on the highest permittivity value; (c) of Fig. 14(c), (d) with multiplicative smoothing. The VP values are indicated as the intersections of the solid horizontal line and the solid vertical lines and the exact permittivity values are indicated with dashed lines.

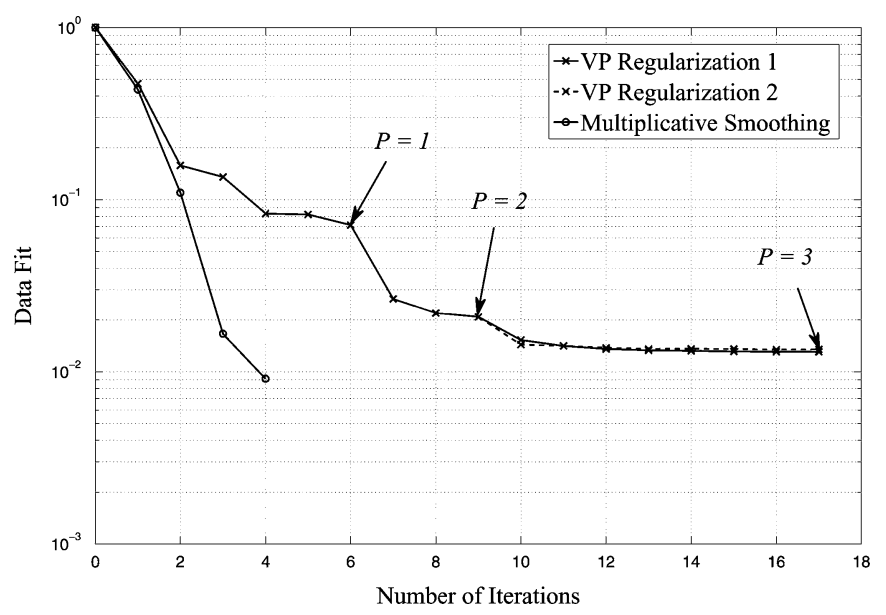

Fig. 13. The data fit cost function $\mathcal{F}^{\mathrm{LS}}$ versus the number of iterations for the reconstructions from measured data using VP regularization with real VP values (VP regularization 1), using VP regularization with real VP values and a lower bound of 2 on the largest VP value (VP regularization 2) and using multiplicative smoothing regularization. The end of each step in the SRVP regularization scheme is indicated with an arrow. An extra VP value is added when $\left\|\boldsymbol{g}^{Q}\right\| \leq \sqrt{2} \cdot 10^{-4}$ or when the data fit cost function $\mathcal{F}^{\mathrm{LS}}$ increases again.

The additional bound thus is not restrictive in the final reconstruction, since the corresponding VP value has moved away from it. Considering only the data fit and the amount of clustering in the permittivity values, the reconstructions of Figs. 11 and 14(a), (b) are hardly distinguishable. In the second reconstruction, however, the permittivities are much better estimated, although the inner cube is somewhat smaller.

Finally, again for comparison, a reconstruction with multiplicative smoothing regularization is shown in Fig. 14(c) and (d). The noise level is reached in only 4 iterations as can be seen in Fig. 13, but the result is not as good as with VP regularization. The overall structure of the target is 


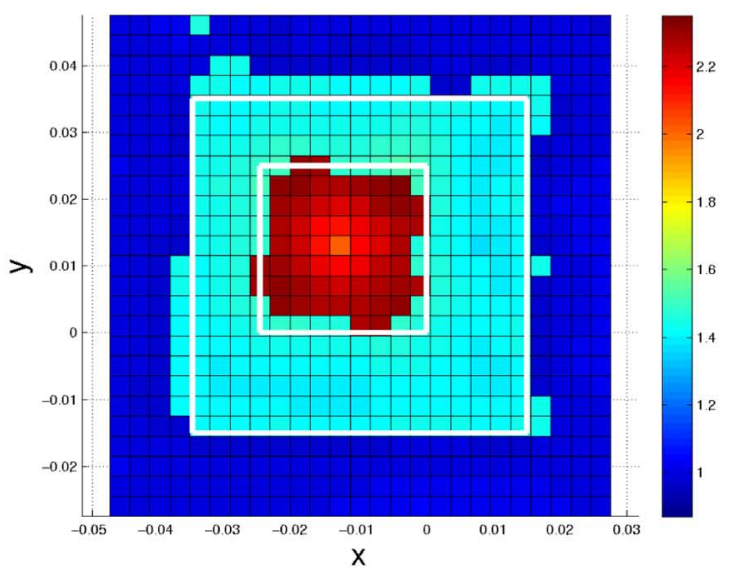

(a)

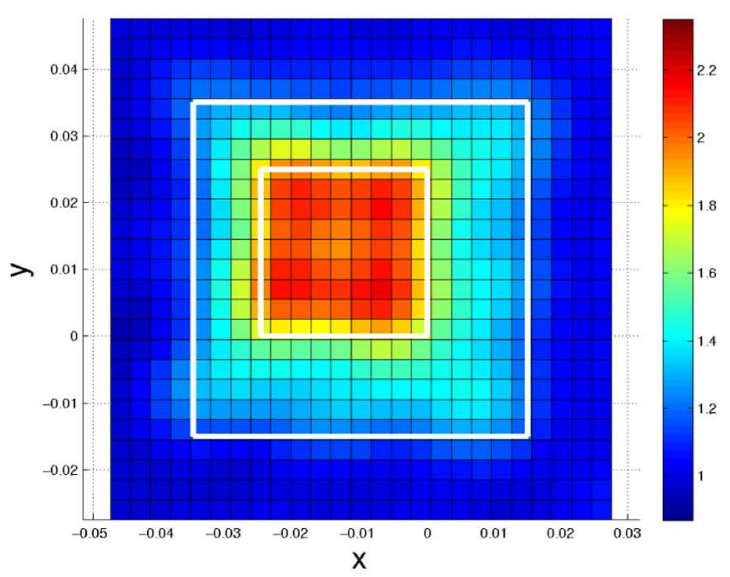

(c)

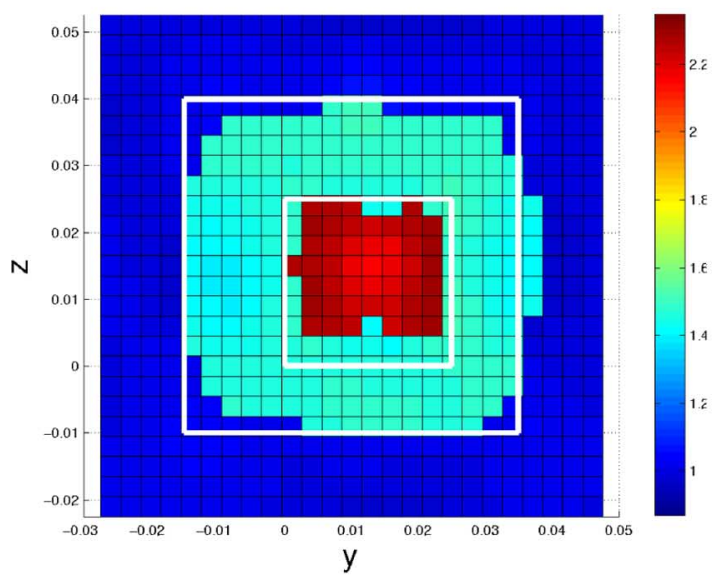

(b)

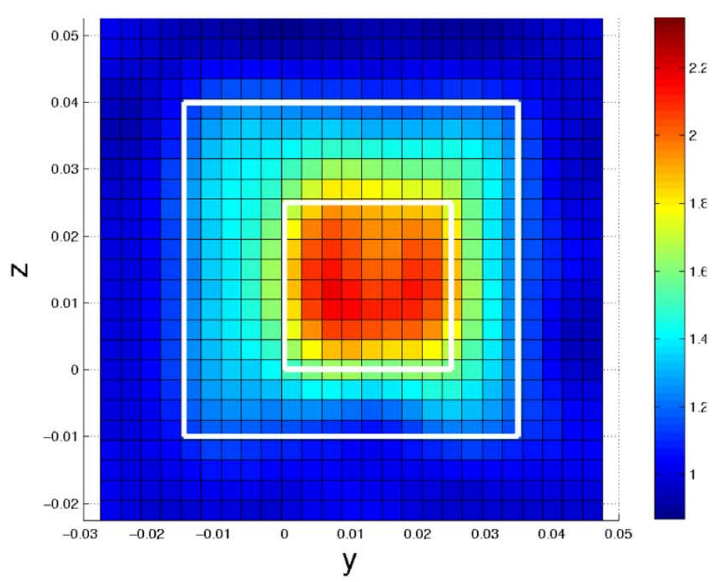

(d)

Fig. 14. Reconstruction at $8 \mathrm{GHz}$ with SRVP regularization $(\gamma=5.0$ and real VP values) of the target of Fig. 9 with an additional lower bound of 2 for the highest permittivity value (a), (b). Reconstruction with multiplicative smoothing regularization of the same target (c), (d). The initial guess is $\epsilon=\epsilon_{0}$ everywhere. Real part of the permittivity in two orthogonal cuts through the center of the investigation domain.

present, but due to the smoothness of the reconstruction, the dimensions of the cubes as well as their permittivity cannot be easily estimated. This becomes very apparent in a swarm plot [Fig. 12(c)], where no clusters of permittivity values can be detected. Moreover, the imaginary parts are spread out too far from the real axis.

\section{CONCLUSION}

In this paper, a new value picking regularization was presented. When applied to the electromagnetic inverse scattering problem, it favors permittivity profiles which consist of a limited number of different permittivity values. For piecewise homogeneous objects, the reconstructions obtained with the new method are superior to reconstructions with a smoothing regularization, because the a priori information hidden in the regularizing function is more appropriate for such targets. Moreover, the VP cost function is simply used as an additive penalty term in the total cost function of the inverse solver and the proposed half quadratic minimization is easily incorporated in a Gauss-Newton optimization framework. Reconstructions from both simulated and measured data have demonstrated the applicability of the proposed algorithm to realistic inverse scattering problems and the high quality of the resulting images. It should be noted finally that VP regularization might have applications in other inverse problems where the unknown model parameters should only take on a limited number of different values.

\section{APPENDIX}

In addition to the choice function $f^{P}$, defined in (6)-(8), we introduce the function $G^{P}$, defined by the recursion

$$
\begin{aligned}
G^{P}\left(u_{1}, \ldots, u_{P} ; x\right)= & \frac{1}{u_{P}+x}+G^{P-1}\left(u_{1}, \ldots, u_{P-1} ; x\right) \\
& -G^{P-1}\left(u_{1}, \ldots, u_{P-1} ; x+u_{P}\right)
\end{aligned}
$$

with

$$
G^{1}\left(u_{1} ; x\right)=\frac{1}{u_{1}+x} .
$$

This function is not defined for $x=0$ whenever one of the arguments $u_{p}$ is zero.

The following properties can be proven [29]:

1) $f^{P}$ is a fully symmetric function, i.e., it is invariant under arbitrary permutations of its arguments. This allows one to 
rewrite $f^{P}\left(u_{1}, \ldots, u_{P}\right)$ as $f^{P}\left(u_{\{P\}}\right)$, where $\{P\}$ is short for $\{1, \ldots, P\}$ and $u_{\{P\}}$ is the set $\left\{u_{p}: p \in\{P\}\right\}$.

2) $f^{P}=0$ if and only if at least one of its arguments is zero.

3) $f^{P}\left(c u_{1}, \ldots, c u_{P}\right)=c f^{P}\left(u_{1}, \ldots, u_{P}\right)$.

4) If $k$ of its arguments are much smaller than the rest, the $P$-dimensional choice function reduces to the choice function of dimension $k$, evaluated in the smaller arguments, i.e.

$$
\begin{aligned}
f^{P}\left(u_{\{P\}}\right) \approx f^{k}\left(u_{I}\right), \quad \text { when } u_{i} & \ll u_{p} \\
\forall i \in I, \quad \forall p & \in\{P\} \backslash I
\end{aligned}
$$

where $I$ is a set of $k \leq P$ indices $I \subset\{P\}$.

5) $f^{P}\left(u_{\{P\}}\right) \leq f^{P-1}\left(u_{\{P\} \backslash p}\right), \forall p \in\{P\}$.

6) $G^{P}\left(u_{1}, \ldots, u_{P} ; x\right)$ is invariant under permutations of the arguments $u_{1}, \ldots, u_{P}$ and can, thus, be written as $G^{P}\left(u_{\{P\}} ; x\right)$.

7) The derivatives of $f^{P}$ are given by

$$
\begin{aligned}
& \left(\frac{\partial}{\partial u_{p}} f^{P}\right)\left(u_{1}, \ldots, u_{P}\right) \\
& \quad=f^{P}\left(u_{\{P\}}\right)\left[G^{P}\left(u_{\{P\}} ; 0\right)-G^{P-1}\left(u_{\{P\} \backslash p} ; 0\right)\right] \\
& \quad=B_{p}^{P}\left(u_{1}, \ldots, u_{P}\right), p=1, \ldots, P
\end{aligned}
$$

which introduces the weight functions $B_{p}^{P}$.

8) The weight functions $B_{p}^{P}$ have range $[0,1]$, with

$$
\begin{aligned}
& B_{p}^{P}\left(u_{1}, \ldots, u_{P}\right)=0 \\
& \quad \Leftrightarrow \quad \exists p^{\prime} \neq p: u_{p^{\prime}}=0 \text { and } u_{p} \neq 0 \\
& B_{p}^{P}\left(u_{1}, \ldots, u_{P}\right)=1 \\
& \quad \Leftrightarrow \quad u_{p}=0 \text { and } u_{p^{\prime}} \neq 0, \forall p^{\prime} \neq p .
\end{aligned}
$$

The function $B_{p}^{P}$ is defined everywhere, except in the intersections of the hyperplane $u_{p}=0$ with other hyperplanes $u_{p^{\prime}}=0$. When approaching these intersections, there exists a finite limit value in the interval ] $0,1[$ which depends on the approach path.

9) $B_{p}^{P}$ is invariant under scaling of its arguments, i.e., $B_{p}^{P}\left(c u_{1}, \ldots, c u_{P}\right)=B_{p}^{P}\left(u_{1}, \ldots, u_{P}\right)$.

10) The hyperplane

$$
\begin{aligned}
Q^{P}\left(u_{1}, \ldots, u_{P} ; u_{1,0}, \ldots,\right. & \left.u_{P, 0}\right) \\
& =\sum_{p=1}^{P} B_{p}^{P}\left(u_{1,0}, \ldots, u_{P, 0}\right) u_{p}
\end{aligned}
$$

for fixed $\left(u_{1,0}, \ldots, u_{P, 0}\right)$ touches (i.e., coincides with and has the same gradient vector as) $f^{P}$ in the point $\left(u_{1,0}, \ldots, u_{P, 0}\right)$.

There is furthermore a property which we were unable to prove for general dimension $P$ up till now, but which has been verified on numerical test grids:

11) $Q^{P}\left(u_{1}, \ldots, u_{P} ; u_{1,0}, \ldots, u_{P, 0}\right) \geq f^{P}\left(u_{1}, \ldots, u_{P}\right)$. This can be proven if one can show that $f^{P}$ is a concave function (but not strictly concave) on $\mathbb{R}_{+}^{P}$, which is easily verified in the specific cases $P=1$ and $P=2$.

\section{REFERENCES}

[1] J. Hadamard, "Sur les problemes aux derivees partielles et leur signification physique," Princeton U. Bull., vol. 13, pp. 49-52, 1902.

[2] A. G. Tijhuis, K. Belkebir, and A. C. S. Litman, "Theoretical and computational aspects of 2-D inverse profiling," IEEE Trans. Geosci. Remote Sens., vol. 39, no. 6, pp. 1316-1330, 2001.

[3] A. Abubakar, P. M. van den Berg, and J. J. Mallorqui, "Imaging of biomedical data using a multiplicative regularized contrast source inversion method," IEEE Trans. Microw. Theory Tech., vol. 50, no. 7, pp. 1761-1770, 2002.

[4] A. Franchois and A. G. Tijhuis, "A quasi-Newton reconstruction algorithm for a complex microwave imaging scanner environment," Radio Sci., vol. 38, no. 2, 2003.

[5] J. De Zaeytijd, A. Franchois, C. Eyraud, and J. M. Geffrin, "Full-wave three-dimensional microwave imaging with a regularized Gauss-Newton method-Theory and experiment," IEEE Trans. Antennas Propag., vol. 55, no. 11, 2007.

[6] P. M. van den Berg and R. E. Kleinman, "A total variation enhanced modified gradient algorithm for profile reconstruction," Inverse Problems, vol. 11, pp. L5-L10, 1995.

[7] C. R. Vogel and M. E. Oman, "Fast, robust total variation-based reconstruction of noisy, blurred images," IEEE Trans. Image Process., vol. 7, no. 6, pp. 813-824, 1998.

[8] P. M. van den Berg, A. L. van Broekhoven, and A. Abubakar, "Extended contrast source inversion," Inverse Problems, vol. 15, no. 5, pp. 1325-1344, 1999.

[9] A. Abubakar and P. M. van den Berg, "Total variation as a multiplicative constraint for solving inverse problems," IEEE Trans. Image Process., vol. 10, no. 9, 2001.

[10] P. Charbonnier, L. Blanc-Féraud, G. Aubert, and M. Barlaud, "Deterministic edge-preserving regularization in computed imaging," IEEE Trans. Image Process., vol. 6, no. 2, 1997.

[11] P. Lobel, C. Pichot, L. Blanc-Féraud, and M. Barlaud, "Microwave imaging: Reconstructions from experimental data using conjugate gradient and enhancement by edge-preserving regularization," Int. J. Imag. Syst. Technol., vol. 8, no. 4, pp. 337-342, 1997.

[12] P. Lobel, C. Pichot, L. Blanc-Féraud, and M. Barlaud, "Conjugategradient algorithm with edge-preserving regularization for image reconstruction from Ipswich data for mystery objects," IEEE Antennas Propag. Mag., vol. 39, no. 2, pp. 12-14, 1997.

[13] A. Litman, D. Lesselier, and F. Santosa, "Reconstruction of a two-dimensional binary obstacle by controlled evolution of a level-set," Inverse Problems, vol. 14, pp. 685-706, 1998.

[14] A. Litman, "Reconstruction by level sets of n-ary scattering obstacles," Inverse Problems, vol. 21, pp. S131-S152, 2005.

[15] D. Colton and P. Monk, "A modified dual space method for solving the electromagnetic inverse scattering problem for an infinite cylinder," Inverse Problems, vol. 10, pp. 87-107, 1994.

[16] L. Crocco and T. Isernia, "Inverse scattering with real data: Detecting and imaging homogeneous dielectric objects," Inverse Problems, vol. 17, no. 6, pp. 1573-1583, 2001.

[17] A. Abubakar and P. M. van den Berg, "The contrast source inversion method for location and shape reconstructions," Inverse Problems, vol. 18, pp. 495-510, 2002.

[18] J. De Zaeytijd, I. Bogaert, and A. Franchois, "An efficient hybrid MLFMA-FFT solver for the volume integral equation in case of sparse 3-D inhomogeneous dielectric scatterers," J. Comput. Phys., vol. 227, no. 14, pp. 7052-7068, 2008.

[19] O. M. Bucci, C. Gennarelli, and C. Savarese, "Representation of electromagnetic fields over arbitrary surfaces by a finite and nonredundant number of samples," IEEE Trans. Antennas Propag., vol. 46, no. 3, pp. 351-359, 1998.

[20] R. Fletcher, Practical Methods of Optimization, 2nd ed. New York: Wiley, 1990.

[21] T. M. Habashy and A. Abubakar, "A general framework for constraint minimization for the inversion of electromagnetic measurements," Progress in Electromagn. Res., vol. 46, pp. 265-312, 2004.

[22] N. Joachimowicz, C. Pichot, and J. Hugonin, "Inverse scattering: An iterative numerical method for electromagnetic imaging," IEEE Trans. Antennas Propag., vol. 39, no. 12, pp. 1742-1752, 1991.

[23] W. C. Chew and Y. M. Wang, "Reconstruction of two-dimensional permittivity distribution using the distorted born iterative method," IEEE Trans. Med. Imag., vol. 9, no. 2, 1990.

[24] A. Franchois and C. Pichot, "Microwave imaging-Complex permittivity reconstruction with a Levenberg-Marquardt method," IEEE Trans. Antennas Propag., vol. 45, no. 2, pp. 203-215, 1997. 
[25] A. N. Tikhonov and V. Y. Arsenine, Solutions of Ill-Posed Problems. Wash. DC: Winston, 1977.

[26] J. M. Geffrin, P. Sabouroux, and C. Eyraud, "Free space experimental scattering database continuation: Experimental set-up and measurement precision," Inverse Problems, vol. 21, pp. S117-S130, 2005.

[27] C. Eyraud, J. M. Geffrin, P. Sabouroux, P. Chaumet, H. Tortel, H. Giovannini, and A. Litman, "Validation of a 3D bistatic microwave scattering measurement setup," Radio Sci., vol. 43, RS4018, doi:10.1029/ 2008RS003836.

[28] J. M. Geffrin, P. Chaumet, C. Eyraud, K. Belkebir, and P. Sabouroux, "Electromagnetic three-dimensional reconstruction of targets from free space experimental data," Appl. Phys. Lett., vol. 92, p. 194102, 2008.

[29] J. De Zaeytijd, "On the 3D electromagnetic quantitative inverse scattering problem: Algorithms and regularization," Ph.D. dissertation, Ghent Univ., Ghent, Belgium, in preparation..

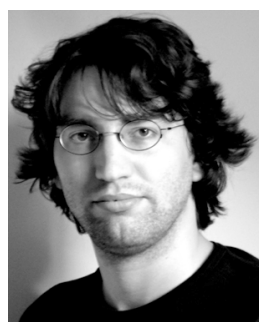

Jürgen De Zaeytijd was born in Oudenaarde, Belgium, in 1981. He received the B.Sc. and M.Sc. degrees (in physical engineering) from Ghent University, Belgium, in 2004. His Masters thesis dealt with the simulation of electromagnetic scattering from electrically large two-dimensional scatterers by means of the fast multipole method.

In October 2004, he joined the Electromagnetics Group of the Department of Information Technology (INTEC), Ghent University. His research, which is funded by a FWO doctoral grant, is focused on the exact inverse scattering problem using microwaves.

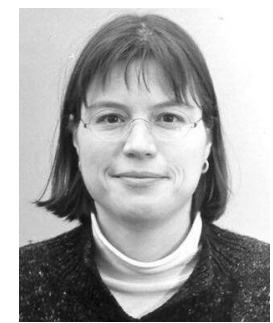

Ann Franchois (M'96-SM'99) was born in Geel, Belgium, in 1964. She received the M.S. degree in electrical engineering from the Ghent University, Belgium, in 1988, and the DEA and Ph.D. degrees in optics and photonics from the University of Paris-Sud, Orsay, France, in 1989 and 1993, respectively.

From 1994 to 1996, she was with the Research Division of Alcatel Bell Telephone, Antwerp, Belgium, working on EMC and from 1996 to 1999, she was with the Joint Research Center of the Commission of the European Communities, Ispra, Italy, working on microwave sensing applications. From 1999 to 2000, she held a Postdoctoral teaching-research position with the Electromagnetics Group, Faculty of Electrical Engineering, Eindhoven University of Technology, The Netherlands. In 2000, she joined the Department of Information Technology (INTEC-IMEC), Ghent University, where she is an Associate Professor. Her research interests include inverse scattering and imaging.

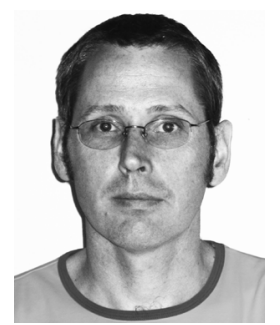

Jean-Michel Geffrin received the Ph.D. degree in physics from the University of Paris XI, France, in 1993.

He then worked for 10 years as a Research Engineer with the Laboratoire des Signaux et Systèmes, Gif-sur-Yvette, France, where he developed specific antennas and experimental setups for measuring targets scattering pattern. In 2002, he joined the Institut Fresnel, Marseille, France, to reinforce the hyperfrequency experimentalist team and has contributed to the constitution of the second and third databases of scattered fields proposed by the Institut Fresnel to the inverse problem community. 